\title{
Boson stars driven to the brink of black hole formation
}

\author{
Scott H. Hawley ${ }^{*}$ and Matthew W. Choptuik ${ }^{1,2} \dagger$ \\ ${ }^{1}$ Center for Relativity, Department of Physics, University of Texas at Austin, Austin, TX 78712-1081 USA \\ ${ }^{2}$ CIAR Gravity and Cosmology Program, Department of Physics and Astronomy, University of British Columbia, Vancouver, \\ British Columbia, Canada V6T $1 Z 1$
}

\begin{abstract}
We present a study of black hole threshold phenomena for a self-gravitating, massive complex scalar field in spherical symmetry. We construct Type I critical solutions dynamically by tuning a one-parameter family of initial data consisting of a boson star and a massless real scalar field. The massless field is used to perturb the boson star via a purely gravitational interaction which results in a significant transfer of energy from the massless field to the massive field. The resulting (unstable) critical solutions, which display great similarity with unstable boson stars, persist for a finite time before either dispersing most of the mass to infinity (leaving a diffuse remnant) or forming a black hole. To further the comparison between our critical solutions and boson stars, we verify and extend the linear stability analysis of Gleiser and Watkins [M. Gleiser and R. Watkins, Nucl. Phys. B319 733 (1989)] by providing a method for calculating the radial dependence of boson star quasinormal modes of nonzero frequency. The frequencies observed in our critical solutions coincide with the mode frequencies obtained from perturbation theory, as do the radial profiles of many of the modes. For critical solutions less than $90 \%$ of the maximum boson star mass $M_{\max } \simeq 0.633 M_{P l}^{2} / m$, the existence of a small halo of matter in the tail of the solution distorts the profiles which otherwise agree very well with unstable boson stars. These halos appear to be artifacts of the collision between the original boson star and the massless field, and do not appear to belong to the true critical solutions, which are interior to the halos and which do in fact correspond to unstable boson stars. It appears that unstable boson stars are unstable to dispersal ("explosion") in addition to black hole formation, and given the similarities in macroscopic stability between boson stars and neutron stars, we suggest that those neutron star configurations at or beyond the point of instability may likewise be unstable to explosion.
\end{abstract}

PACS number(s): 04.40.-b, 04.25.Dm, 04.40.Dg

\section{INTRODUCTION}

Over the past decade, detailed studies of models of gravitational collapse have revealed that the threshold of black hole formation is generically characterized by special, "critical" solutions. The features of these solutions are known as "critical phenomena," and arise in even the simplest collapse models, such as a model consisting of a single, real, massless scalar field, minimally coupled to the general relativistic field in spherical symmetry [i]. Although we present a brief overview of black hole critical phenomena here, we suggest that interested readers consult the excellent reviews by Gundlach [2,3] for many additional details.

Black hole critical solutions can be constructed dynamically via simulation, i.e. via solution of the full timedependent PDEs describing the particular model, by considering one-parameter families of initial data which are required to have the following "interpolating" property: for sufficiently large values of the family parameter, $p$, the evolved data describes a spacetime containing a black hole, whereas for sufficiently small values of $p$, the matter-energy in the spacetime disperses to large radii at late times, and no black hole forms. For any such family, there will exist a critical parameter value, $p=p^{\star}$, which demarks the onset, or threshold, of black hole formation. To date at least, it has invariably turned out that the solutions which appear in the strongly-coupled regime of the calculations (i.e. the critical solution), are almost totally independent of the specifics of the particular family used as a generator. In fact, the only initial-data dependence which has been observed so far in critical collapse occurs in models for which there is more than one distinct black-hole-threshold solution. In this sense then, black hole critical solutions are akin to, for example, the Schwarzschild solution, which can be formed through the collapse of virtually any type and/or shape of spherically distributed matter. In particular, like the Schwarzschild solution, black hole critical solutions possess additional symmetry (beyond spherical symmetry) which, to date, has either been a time-translation symmetry, in

\footnotetext{
${ }^{*}$ E-mail address: shawley @ physics.utexas.edu
}

${ }^{\dagger}$ E-mail address: choptuik@ physics.ubc.ca 
which the critical solution is static or periodic, or a scale-translation symmetry (hometheticity), in which the critical solution is either continuously or discretely self-similar (CSS or DSS).

However, in clear contrast to the Schwarzschild solution, black hole threshold solutions are, by construction, unstable.

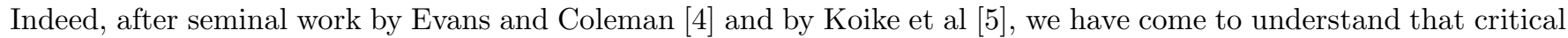
solutions are in some sense minimally unstable, in that they tend to have precisely one unstable mode in linear perturbation theory. Thus letting $p \rightarrow p^{\star}$ amounts to minimizing or "tuning away" the initial amplitude of the unstable mode present in the system.

As already suggested, two principal types of critical behavior have been seen in black hole threshold studies; which type is observed depends, in general, upon the type of matter model and the initial data used - as mentioned, some models exhibit both types of behavior. Historically, one of us termed these Type I and Type II solutions, in a loose analogy to phase transitions in statistical mechanics, but at least at this juncture, we could equally well label the critical solutions by their symmetries (i.e. static/periodic or CSS/DSS) . For Type I solutions, there is a finite minimum black hole mass which can be formed, and, in accord with their static/periodic nature, there is a scaling

law, $\tau \sim-\gamma \ln \left|p-p^{\star}\right|$, relating the lifetime, $\tau$, of a near-critical solution to the proximity of the solution to the critical point. Here $\gamma$ is a model-specific exponent which is the reciprocal of the real part of the eigenvalue associated with the unstable mode. On the other hand, Type II critical behavior - less relevant to the current study - is characterized by arbitrarily small black hole mass at threshold, and critical solutions which are generically self-similar.

The direct construction, or simulation, of critical solutions, has thus far been performed almost exclusively within the ansatz of spherical symmetry. In the spherical case one must couple to at least one matter field for non-trivial dynamics, and spherically symmetric critical solutions for a considerable variety of models have now been constructed and analyzed. In addition to the massless scalar case mentioned above, these include solutions containing a perfect fluid [ [.,6], a scalar non-Abelian gauge field [7], and particularly germane to the current work, a massive real scalar field [8]. The work of Abrahams and Evans [9], which considered axisymmetric critical collapse of gravitational waves, remains notable for being the only instance of a reasonably well-resolved non-spherical critical solution [10].

Our current interest is a critical-phenomena-inspired study of the dynamics associated with "boson stars" [11 13, a class of equilibrium solutions to the Einstein-Klein Gordon system for massive complex fields, which are supported against gravitational collapse by the effective pressure due to the dispersive nature of a massive Klein-Gordon field. (For extensive reviews on the subject of boson stars, see Jetzer [14] or Mielke and Schunck [15].) We know from the studies by Gleiser and Watkins [16] and by Lee and Pang [17], that there exists a critical value of the central density which marks the transition between boson stars which are stable with respect to infinitesimal radial perturbations, and those which are unstable. The dynamical simulations of Seidel and Suen [18] revealed scenarios in which a boson star on the unstable branch would either form a black hole or radiate scalar material and form a boson star on the stable branch. Their study is extended in this paper, in which we consider dynamical changes to the geometry of a boson star which are large enough to bring it to the threshold of black hole formation.

As already mentioned, another paper closely related to this work is that of Brady et al. [8], which described a dynamical study of critical solutions of a massive real scalar field. Those authors demonstrated scenarios in which black holes could be formed with arbitrarily small mass (Type II transitions), and those in which the black holes formed had a finite minimum mass (Type I transitions). The boundary between these regimes seemed to be the relative length scale of the pulse of initial data compared to the Compton wavelength associated with the boson mass. Initial data which was "kinetic energy dominated" evolved in a manner essentially similar to the evolution of a massless scalar field. Initial data pulses having widths larger than the length scale set by the boson mass were "potential dominated," providing a characteristic scale for the formation of the critical solutions. Brady et al. found that the resulting Type I critical solutions corresponded to a class of equilibrium solutions discovered by Seidel and Suen [19], which are called "oscillating soliton stars." These soliton stars share many characteristic with the complex-valued boson stars, such as the relationship between the radius and mass of the star. Both types of "stars" have a maximum mass, and show the same overall behavior as "real" (fermion) stars in terms of the turn-over in their respective stability curves. Interestingly, although the soliton stars are not static - they are periodic (or quasi-periodic) - many of the same macroscopic properties seen in fluid stars are still observed.

In this paper, we construct critical solutions of the Einstein equations coupled to a massive, complex scalar field dynamically, by simulating the implosion of a spherical shell of massless real scalar field around an "enclosed" boson star. The massless field implodes toward the boson star and the two fields undergo a (purely gravitational) "collision." The massless pulse then passes through the origin, explodes and continues to $r \rightarrow \infty$, while the massive complex (boson star) field is compressed into a state which ultimately either forms a black hole or disperses. We can thus play the "interpolation game" using initial data which result in black hole formation, and initial data which give rise to dispersal: specifically, we vary the initial amplitude of the massless pulse to tune to a critical solution. We analyze the black hole threshold solutions obtained in this manner, and discuss the similarities between our critical solutions for the self-gravitating complex massive scalar field and boson stars on the unstable branch. To further this discussion, we extend the work of Gleiser and Watkins [16] and compare the results of the simulations with those of 
linear perturbation theory.

The layout of the remainder this paper is as follows: In Section II, we describe the mathematical basis for our numerical simulations. In Section III, we present results from our simulations, in which the Type I character of the critical solutions is demonstrated, along with the close similarities one finds between the features of the critical solutions and those of boson stars. In most of the critical solutions we find a halo of mass near the outer edge of the solution which is not a feature of boson star equilibrium data. Inside this halo, however, the critical solutions match the boson star profiles very well. In Section IV, we give a synopsis of our linear stability analysis of boson star quasinormal modes, from which we obtain the boson star mode frequencies as functions of the central value of the modulus of the complex field. Section V concerns the radial profiles of the perturbative modes per se, and includes a comparison of the mode shapes and frequencies obtained from perturbation theory with our simulation data. The modes obtained by these two different methods agree well with each other, although the additional oscillatory mode in our simulation data is only shown to agree with the corresponding boson star mode in terms of the oscillations in the metric and not in the field (possibly as an artifact of our simplistic approach to extracting this mode from the simulation). In Section V we provide further discussion regarding the properties of the halos surrounding the critical solutions.

Conclusions in Section VI are followed by appendices giving tables of mode frequencies versus the central field value of the boson star, details about our finite difference code, and details of our linear stability analysis, which includes a description of our algorithm for finding the frequencies of boson star modes.

\section{SCALAR FIELD MODEL}

A boson star is described by a complex massive scalar field $\phi$, minimally coupled to gravity as given by general relativity. We work solely within the context of classical field theory, and choose units in which $G$ and $c$ are unity. Furthermore, since all lengths in the problem can be scaled by the boson mass $m$ [13], we choose $m=1$. To the usual boson star model, we add an additional, massless real scalar field, $\phi_{3}$, which is also minimally coupled to gravity. This additional scalar field will be used to dynamically "perturb" the boson star.

The equations of motion for the system are then the Einstein equation and Klein-Gordon equations:

$$
\begin{gathered}
G_{a b}=R_{a b}-\frac{1}{2} g_{a b} R=8 \pi\left(T_{a b}^{C}(\phi)+T_{a b}^{R}\left(\phi_{3}\right)\right) \\
\square \phi-m^{2} \phi=0 \\
\square \phi_{3}=0
\end{gathered}
$$

where

$$
\begin{gathered}
8 \pi T_{a b}^{C}(\phi)=\partial_{a} \phi \partial_{b} \phi^{*}+\partial_{a} \phi^{*} \partial_{b} \phi-g_{a b}\left(\partial_{c} \phi \partial^{c} \phi^{*}+m^{2}|\phi|^{2}\right), \\
8 \pi T_{a b}^{R}\left(\phi_{3}\right)=2 \partial_{a} \phi_{3} \partial_{b} \phi_{3}-g_{a b} \partial^{c} \phi_{3} \partial_{c} \phi_{3},
\end{gathered}
$$

and $\square$ is the D'Alembertian operator. While more general potentials in (2.2) have been employed recently [20,21], we will restrict our discussion to the simplest case, i.e. merely the $m^{2} \phi^{2}$ potential. We also stress that the complex scalar field, $\phi$, and the massless, real scalar field, $\phi_{3}$ are coupled only through gravity - in particular we do not include any interaction potential $V_{I}\left(\phi, \phi_{3}\right)$.

Restricting our attention to spherical symmetry, we write the most general spherically-symmetric metric using Schwarzschild-like "polar-areal" coordinates

$$
d s^{2}=-\alpha^{2}(t, r) d t^{2}+a^{2}(t, r) d r^{2}+r^{2} d \Omega^{2},
$$

and generally make use of the " $3+1$ " formalism of Arnowitt, Deser and Misner [22] which regards spacetime as a foliation of spacelike hypersurfaces parameterized by $t$.

We write the (spherically-symmetric) complex field, $\phi(t, r)$, in terms of its components

$$
\phi(t, r)=\phi_{1}(t, r)+i \phi_{2}(t, r)
$$


where $\phi_{1}(t, r)$ and $\phi_{2}(t, r)$ are each real. Again, since our model includes no self-interaction (anharmonic) potential for the complex field, $\phi_{1}$ and $\phi_{2}$ are only coupled through the gravitational field.

We then define

$$
\begin{array}{cc}
\Phi_{1}(t, r) \equiv \phi_{1}^{\prime} & \Phi_{2}(t, r) \equiv \phi_{2}^{\prime} \\
\Pi_{1}(t, r) \equiv \frac{a}{\alpha} \dot{\phi}_{1} & \Pi_{2}(t, r) \equiv \frac{a}{\alpha} \dot{\phi}_{2}, \\
\Phi_{3}(t, r)=\phi_{3}^{\prime} & \Pi_{3}(t, r)=\frac{a}{\alpha} \dot{\phi_{3} .}
\end{array}
$$

where $^{\prime} \equiv \partial / \partial r$ and ${ }^{`} \equiv \partial / \partial t$.

With these definitions, the equations we solve are the Hamiltonian constraint,

$$
\frac{a^{\prime}}{a}=\frac{1-a^{2}}{2 r}+\frac{r}{2}\left[\Pi_{1}^{2}+\Pi_{2}^{2}+\Pi_{3}^{2}+{\Phi_{1}}^{2}+{\Phi_{2}}^{2}+\Phi_{3}{ }^{2}+a\left({\phi_{1}}^{2}+{\phi_{2}}^{2}\right)\right],
$$

(where $\Pi_{1}^{2}$ should be read as $\left(\Pi_{1}\right)^{2}$ ), the slicing condition,

$$
\frac{\alpha^{\prime}}{\alpha}=\frac{a^{2}-1}{r}+\frac{a^{\prime}}{a}-a^{2} r\left(\phi_{1}^{2}+\phi_{2}^{2}\right)
$$

and the Klein-Gordon equations,

$$
\dot{\Pi}_{k}=3 \frac{\partial}{\partial r^{3}}\left(\frac{r^{2} \alpha}{a} \Phi_{k}\right)^{\prime}-\alpha a \phi_{k}\left(1-\delta_{3 k}\right),
$$

where $k=1,2,3$ and $\delta_{3 k}$ is a Kronecker delta used to denote the fact that $\phi_{3}$ is a massless field.

We also have equations which are used to update the spatial gradients of the scalar fields, as well as the scalar fields themselves. These follow directly from the definitions (2.8) and (2.9):

$$
\begin{aligned}
\dot{\Phi}_{k} & =\left(\frac{\alpha}{a} \Pi_{k}\right)^{\prime} \\
\phi_{k} & =\int_{0}^{r} \Phi_{k} d \tilde{r}
\end{aligned}
$$

Equations (2.11)-(2.15) are solved numerically using the second order finite difference method described in Appendix B.

Initial conditions for our simulations are set up as follows. First, initial data for the massive field are constructed from the boson star ansatz

$$
\phi(t, r)=\phi_{0}(r) e^{-i \omega t},
$$

where we let $\phi_{0}(r)$ be real. Substitution of this ansatz into the full set of equations (2.11)-(2.15), yields a system of ordinary differential equations (ODEs), whose solution, for a given value of the central field modulus, is found by "shooting," as described in [12]. Once the boson star data is in hand, we add the perturbing massless field by freely specifying $\Phi_{3}$ and $\Pi_{3}$. At this point, all matter quantities have been specified ; the initial geometry, $a(0, r)$ and $\alpha(0, r)$ is then fixed by the constraint and slicing equations (2.11) and (2.12).

In relating the simulation results which follow, it is useful to consider the individual contributions of the complex and real fields to the total mass distribution of the space-time, in order that we can meaningfully and unambiguously discuss, for example, the exchange of mass-energy from the real, massless field to the massive, complex field. By Birchoff's theorem, in any vacuum region, the mass enclosed by a sphere of radius $r$ at a given time $t$ is given by the Schwarzschild-like mass aspect function $M(t, r)=r\left(1-1 / a^{2}\right) / 2$. However, at locations occupied by matter, $M(t, r)$ cannot necessarily be usefully interpreted as a "physical" mass. In polar-areal coordinates, the mass aspect function is related to the local energy density $\rho(t, r)$ by

$$
\frac{\partial M(t, r)}{\partial r}=r^{2} \rho(t, r)
$$


with $\rho(t, r)$ given in our case by

$$
\rho(t, r)=\frac{1}{2 a^{2}}\left[\Pi_{1}{ }^{2}+\Pi_{2}^{2}+\Phi_{1}{ }^{2}+{\Phi_{2}}^{2}+a^{2}\left(\phi_{1}{ }^{2}+\phi_{2}{ }^{2}\right)\right]+\frac{1}{2 a^{2}}\left[\Pi_{3}{ }^{2}++\Phi_{3}{ }^{2}\right] .
$$

Here, we have explicitly separated the contributions from the complex and real fields. Since $\partial M / \partial r$ is given by a linear combination of the contributions from each field, we can decompose $\partial M / \partial r$ so that, in instances where there is no overlap in the supports of the distinct fields, we can unambiguously refer to the mass due to one field or the other. That is, we can refer to the individual contributions of each field to the total mass as being physically meaningful masses in their own rights. Then, by integrating the contribution of each field to $\partial M / \partial r$ over some range of radius $\left(r_{\min } \cdots r_{\max }\right)$, (where there is some region of vacuum starting at $r_{\min }$ and extending inward, and some region of vacuum starting at $r>=r_{\max }$ and extending outward), and demanding that none of the other type of field is present in the domain of integration, we can obtain a measure of the mass due to each field.

Motivated by such considerations, we define an energy density for the complex field, $\rho_{C}$, as

$$
\rho_{C}(t, r)=\frac{1}{2 a^{2}}\left[\Pi_{1}^{2}+\Pi_{2}^{2}+\Phi_{1}^{2}+\Phi_{2}^{2}+a^{2}\left(\phi_{1}^{2}+\phi_{2}{ }^{2}\right)\right]
$$

with a corresponding mass aspect function, $M_{C}(t, r)$, given by

$$
M_{C}(t, r)=\int_{0}^{r} \tilde{r}^{2} \rho_{C} d \tilde{r} .
$$

Similarly, the energy density due to the real field is defined as

$$
\rho_{R}(t, r) \equiv \frac{1}{2 a^{2}}\left[\Pi_{3}^{2}++\Phi_{3}^{2}\right]
$$

with a corresponding mass aspect function, $M_{R}(t, r)$ given by

$$
M_{R}(t, r)=\int_{0}^{r} \tilde{r}^{2} \rho_{R} d \tilde{r}
$$

We again emphasize that in regions where the supports of the different fields overlap (and in non-vacuum regions in general) it may not be possible to ascribe physical meaning to the individual mass aspect functions defined above. (However, even in such instances, these functions are still useful diagnostics.) Most importantly, where the supports of the fields do overlap, and only in these regions, it is possible for mass-energy to be exchanged from one scalar field to the other-through the gravitational field - while the sum $M_{C}+M_{R}=M$ (measured in an exterior vacuum region) is conserved. The quantities given above allow us to measure this exchange of mass by looking at the profiles $M_{C}(t, r)$ and $M_{R}(t, r)$ before and after a time when the fields are interacting. This is shown in the next section.

As a further consideration, we point out that the $U(1)$ symmetry of the complex field implies that there is a conserved Noether current, $J^{\mu}$, given by

$$
J^{\mu}=\frac{i}{8 \pi} g^{\mu \nu}\left(\phi \partial_{\nu} \phi^{*}-\phi^{*} \partial_{\nu} \phi\right)
$$

The corresponding conserved charge or "particle number" $N$ is

$$
N=\int_{0}^{\infty} r^{2} \sqrt{-g} J^{t}
$$

We may also wish to regard $N$ as a function of $t$ and $r$ by integrating the above function from zero to some finite radius, in which case

$$
\frac{\partial N(t, r)}{\partial r}=r^{2}\left(\Pi_{1} \phi_{2}-\Pi_{2} \phi_{1}\right) .
$$

Some authors have considered the difference $M_{C}-m N$ to be a sort of "binding energy" of the complex field [14], however this quantity does not correspond to any transition in the stability of boson stars, and we have not found it to be very useful in understanding the dynamics

of our simulations.

Finally, following Seidel and Suen [18], we define a radius $R_{95}(t, r)$ for the boson star implicitly by $\left.M_{C}\right|_{R_{95}}=$ $\left.0.95 M_{C}\right|_{r \rightarrow \infty}$. Alternatively, we will also consider a radius $R_{63}(t, r)$ which encloses $\left(1-e^{-1}\right) \sim 63 \%$ of $\left.M_{C}\right|_{r \rightarrow \infty}$, and which will include the "bulk" of a boson star but will neglect the "tail". 


\section{SIMULATION RESULTS}

We choose the initial data for the complex field to be a boson star at the origin, with a given central density $\phi_{0}(0)$. For the massless field $\phi_{3}(0, r)$, we choose one of the families in Table If. We generate critical solutions by tuning the amplitude $A$ of $\phi_{3}(0, r)$ (holding the position $r_{0}$ and width $\Delta$ constant) using a bisection search, until the resulting solution is arbitrarily close (i.e. within some specified precision) to the point of unstable equilibrium between dispersal and black hole formation.

Figure 1 shows a series of snapshots from a typical simulation in which the parameter $p(p \equiv A)$, is slightly below the critical value $p^{\star}$, for a boson star on the stable branch with a mass of $M=0.59 M_{P l}^{2} / m$ (where $M_{P l}$ is the Planck mass). The shell of massless field, a member of initial data Family I, implodes through the boson star and explodes back out from the origin, and the gravitational interaction between the fields forces the boson star into a new state, a "critical solution." We see from this animation, and from Figure 3, that dispersal from the critical state does not mean that the boson star returns to its original stable configuration, but rather that the star becomes strongly disrupted and "explodes." That is to say, if we were to follow the evolution beyond $t=475$, the massive field would continue to spread toward spatial infinity. At some late time, after a large amount of scalar radiation has been emitted, the end state would probably be a stable boson star with very low mass.

TABLE I. Families of initial data. For both families, the initial data, $\phi(0, r)=\phi_{1}(0, r)+i \phi_{2}(0, r)$, for the massive complex field is given by a boson star, obtained by solving (2.11)-(2.13) numerically according to the ansatz 2.16) (with the parameter $\omega$ found via "shooting"). The initial real massless field profile, $\phi_{3}(0, r)$, is given in closed form by the "gaussian" and "kink" initial data. For each family, we also choose $\partial_{t} \phi_{3}(0, r)$ such that the pulse is initially in-going, ı.e. $\Pi_{3}(0, r)=\Phi_{3}(0, r)+\phi_{3}(0, r) / r$.

\begin{tabular}{c|ccc|ccc}
\hline \hline Family & \multicolumn{3}{|c|}{ Complex Field $\phi_{1}+i \phi_{2}$} & \multicolumn{3}{c}{ Real Field $\phi_{3}$} \\
& Name & Parameters & Profile & Name & Parameters & Profile \\
\hline I & Boson Star & $\phi_{0}(0)$ & $\phi_{0}(r)$ & Gaussian & $A, r_{0}, \Delta$ & $A \exp \left(-\left(\frac{r-r_{0}}{\Delta}\right)^{2}\right)$ \\
II & Boson Star & $\phi_{0}(0)$ & $\phi_{0}(r)$ & Kink & $A, r_{0}, \Delta$ & $\frac{A}{2}\left(1+\tanh \left(\frac{r-r_{0}}{\Delta}\right)\right)$ \\
\hline \hline
\end{tabular}




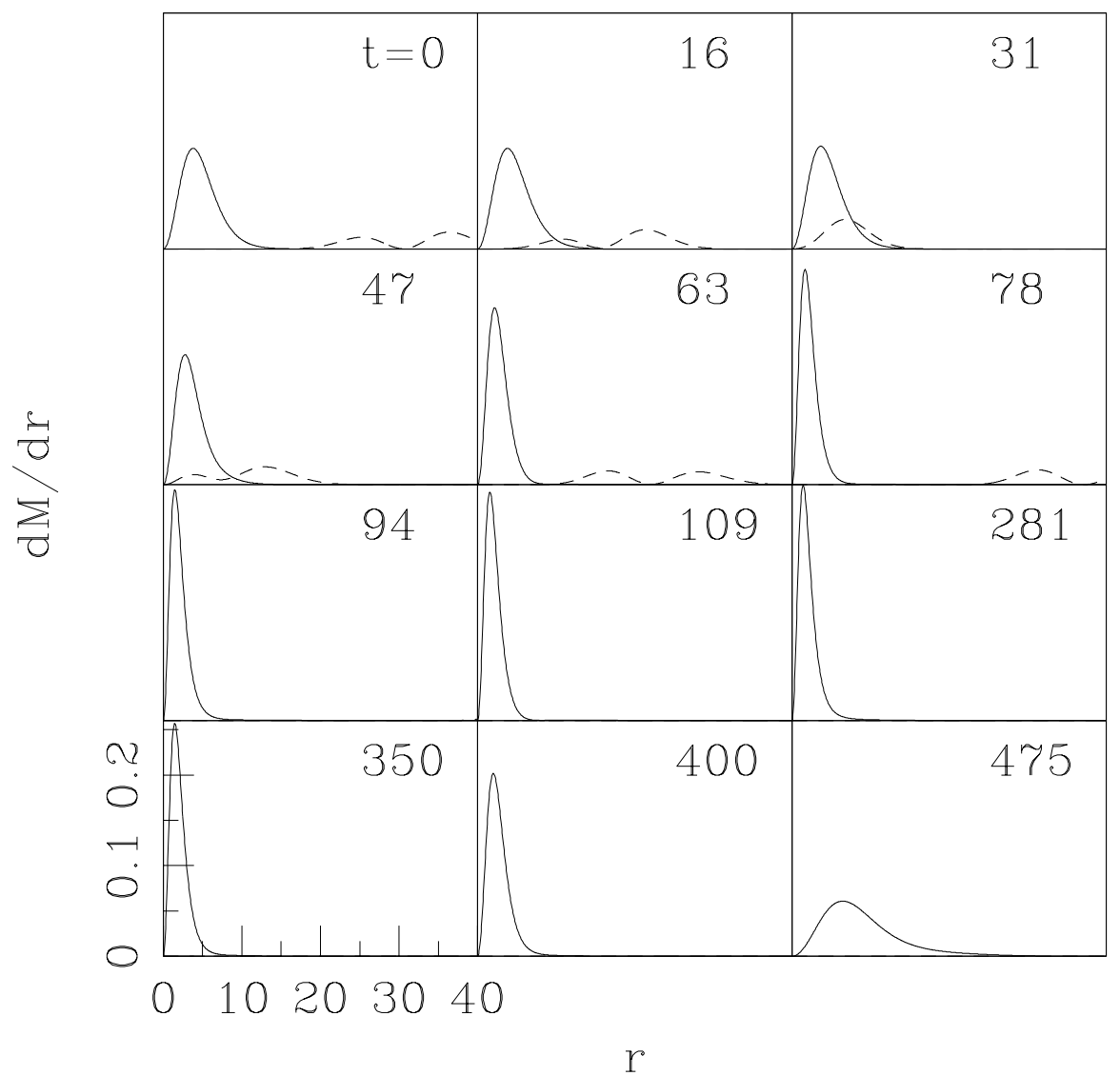

FIG. 1. Evolution of a perturbed boson star with $\phi_{0}(0)=0.04 \times \sqrt{4 \pi}$ and mass $M_{C}=0.59 M_{P l}^{2} / m$. This shows contributions to $\partial M / \partial r$ due to the massive field (solid line) and massless field (dashed line). We start with a stable boson star centered at the origin, and a pulse of massless field given by Family I with $r_{0}=30$ and $\Delta=8$. (We see two peaks in $d M / d r$ of massless field because it is only the gradients of $\phi_{3}$, not $\phi_{3}$ itself, which contribute to $M_{R}(r, t)$ for a massless field.) In the evolution shown above, the pulse of massless field enters the region containing the bulk of the boson star $(t \simeq 15)$, implodes through the origin $(t \simeq 30)$ and leaves the region of the boson star $(t \simeq 50)$. Shortly after the massless pulse passes through the origin, the boson star collapses into a more compact configuration, about which it oscillates for a long time before either forming a black hole or dispersing. (The case of dispersal is shown here.) Note that the perturbing field $\phi_{3}$ passes through the boson star and exits the region containing most of the star, even before the massive field reaches its denser, critical state. Thus the massless field is not part of the critical solution per se. Black hole formation (always with a finite black hole ADM mass in our study) can take place at times long after the massless pulse has left the neighborhood of the boson star.

The gravitational interaction between the two fields results in an exchange of energy from the massless field to the massive field, as shown in Figure 2. Figure 3 shows some timelike slices through the simulation data, giving a plot of the maximum value of $a$, the value of $|\phi|$ at the origin, and the radius $R_{95}$ as functions of time. These are given to help elucidate the point that the critical solution oscillates about some local equilibrium, before dispersing or forming a black hole. The lifetime of the critical solution increases monotonically as $p \rightarrow p^{\star}$. Figure 1 shows that the scaling law expected for Type I transitions is exhibited by these solutions. 


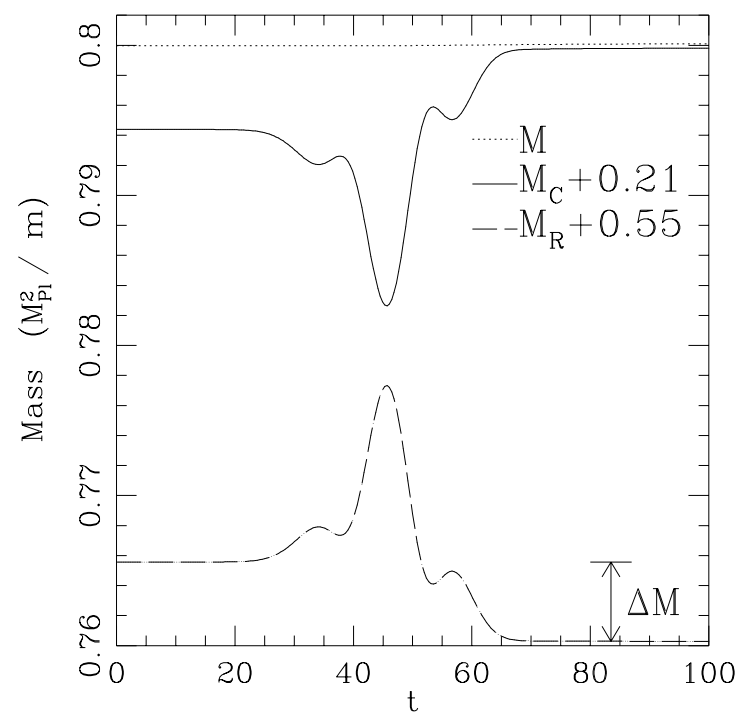

FIG. 2. Exchange of energy between the real and complex scalar fields. For this simulation, initial data from Family I was used, with $\phi_{0}(0)=0.04 \times \sqrt{4 \pi}, r_{0}=40$ and $\Delta=8$. The solid line shows the mass of the complex field, shifted upward on the graph by $0.21 M_{P l}^{2} / m$. The long-dashed line shows the mass of the real field, shifted upward by $0.55 M_{P l}^{2} / m$. The mass $\Delta M$ exchanged from the massless field to the massive field in this simulation is nearly 0.0053 , or about $2.5 \%$ of the mass of the real field ( $9 \%$ of the boson star mass). The amount (and percentage) of mass transfer goes to zero as we consider boson star initial data approaching the transition to instability (see, e.g. Figure 7). The dotted line near the top of the graph shows the total mass enclosed within $r=100$. Throughout the simulation, both the total mass $M=M_{C}+M_{R}$ and the particle number $N$ (of the complex field) are conserved to within a few hundredths of a percent.
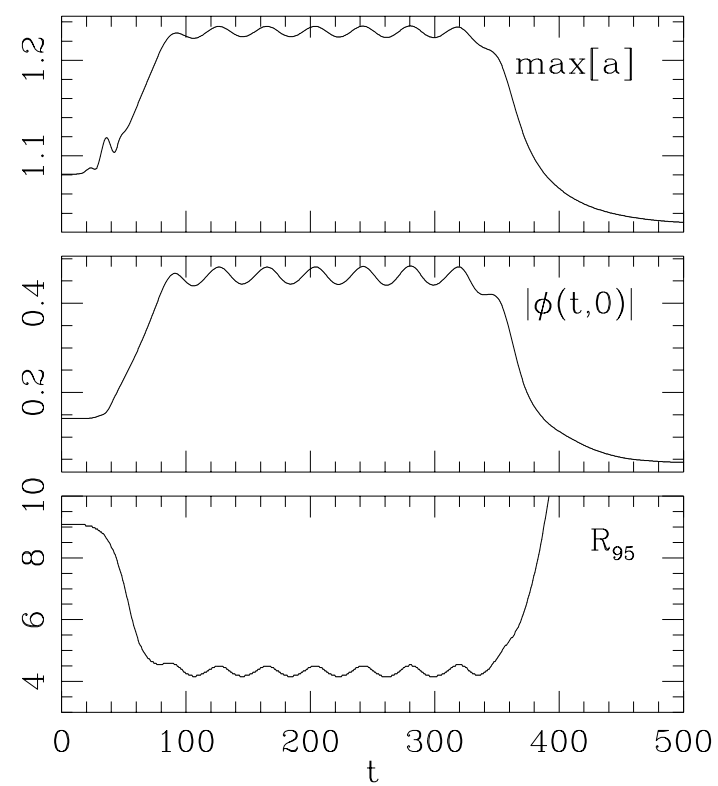

FIG. 3. Quantities describing a near-critical solution. Here we show timelike slices through the data shown in Figure 1, an evolution that ends in dispersal. Top: Maximum value of the metric function $a$ (for each spacelike hypersurface parameterized by $t$ ). The local maximum at $t \simeq 40$ is due to the presence of the pulse of massless field. Middle: Central value $|\phi(t, 0)|$ of the massive field. Bottom: Radius $R_{95}$ which contains $95 \%$ of the mass-energy in the complex field. Again, we see evidence that after the remaining in critical regime for a while, the star can "explode," leaving a diffuse remnant with low mass. 


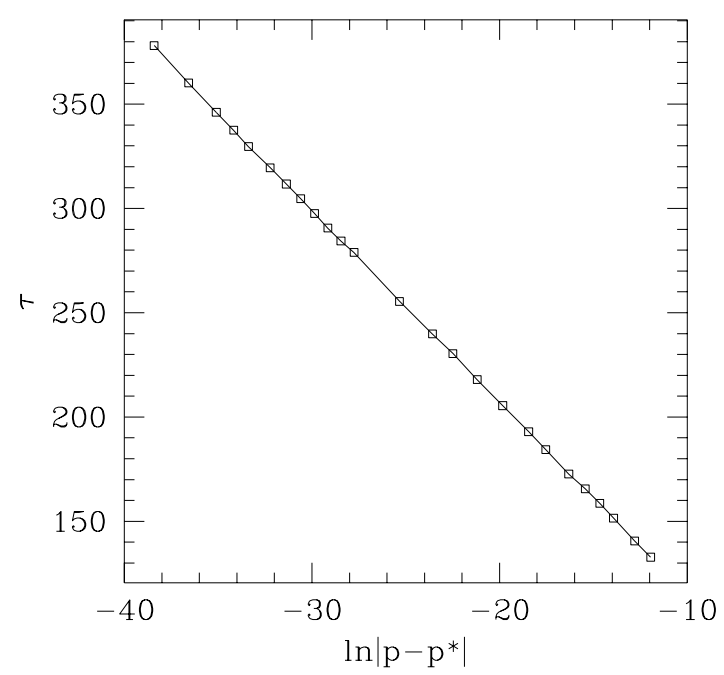

FIG. 4. Lifetime $\tau$ of a typical set of near-critical solutions vs. $\ln \left|p-p^{\star}\right|$. We use initial data from Family I. The lifetime of the critical solution obeys a simple scaling relation. Using super-critical solutions, we measure $\tau$ to be the time from $t=0$ until black hole formation occurs. The relationship shown in the graph can be described by $\tau=-\gamma \ln \left|p-p^{\star}\right|$, where for the data shown in this graph, $\gamma \simeq 9.2$ The value of $\gamma$ can be related to the imaginary part of the Lyapunov exponent $\sigma$ of the unstable mode $\left(\sim e^{i \sigma t}\right)$ by $\Im(\sigma)=1 / \gamma \simeq 0.109$. This value is the same as that obtained from a linear perturbation analysis of the specific boson star to which we believe this configuration is asymptoting (See Section V). We note that in the limit $p \rightarrow p^{\star}$, the mass of the black hole formed is finite (and close to the mass of the progenitive unstable boson star) , i.e. the system exhibits Type I critical behavior.
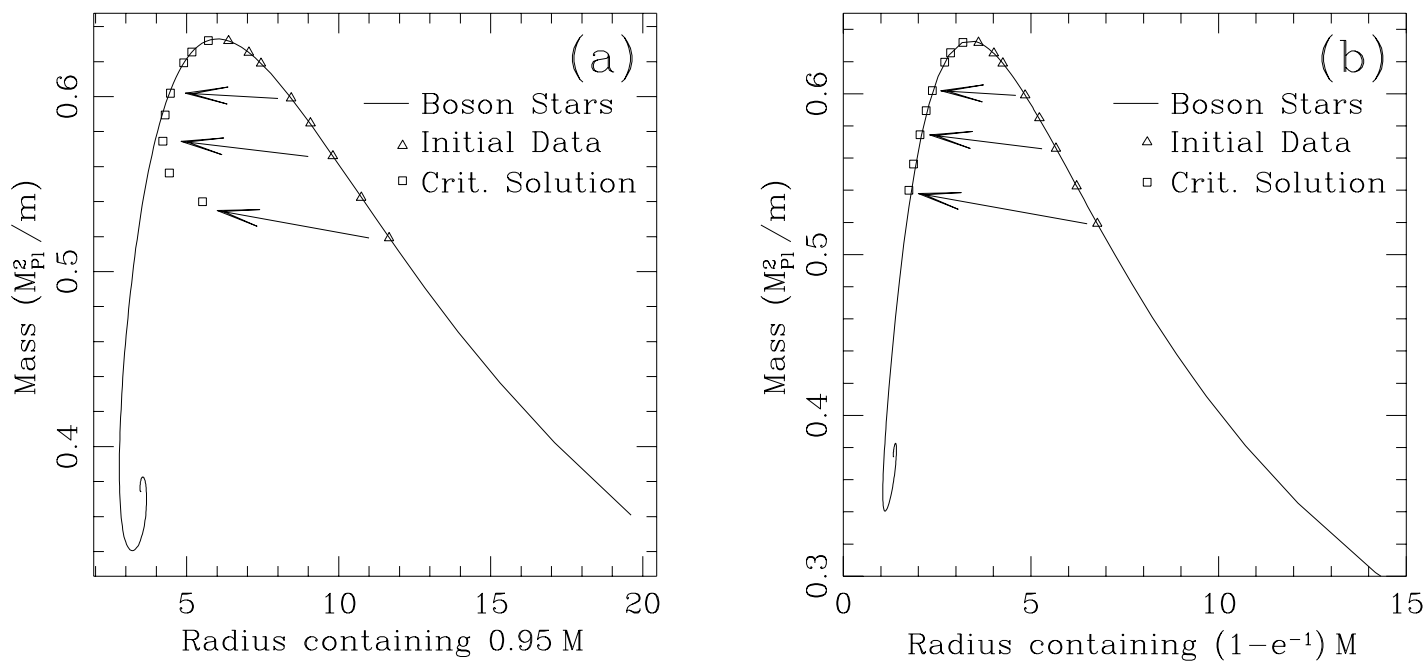

FIG. 5. Mass vs. radius for equilibrium configurations of boson stars (solid line), initial data for the complex field (triangles), and critical solutions (squares). Arrows are given to help match initial data with the resulting critical solutions. Points on the solid line to the left of the maximum mass $M_{\max } \simeq 0.633 M_{P l}^{2} / m$ correspond to unstable boson stars, whereas those to the right of the maximum correspond to stable stars. If one takes time averages of properties such as mass, central density $|\phi(t, 0)|$ and radius $R_{95}$ during the critical regime, one finds values which match the profile of a boson star on the unstable branch. The squares show the time average of each critical solution during the oscillatory phase. Graph (a) shows mass $M$ versus $R_{95}$ the radius containing $95 \%$ of $M$, whereas graph (b) shows $M$ versus the radius containing $\left(1-e^{-1}\right) M$. The agreement between the critical solutions and boson stars shown in graph (a) deteriorates with decreasing mass, however the comparison shown in graph (b), which neglects the "tail" of the critical solutions and boson stars, shows much better agreement for all masses. (We show the tail region in Figure 6.) In this simulation the massive field radiates only a small amount due to the perturbation by the massless field, and so the stable boson star is essentially driven to "pop" across the stability curve by the impinging massless pulse. 
Figure f shows the mass vs. radius for some critical solutions along with the equilibrium curve for boson stars. We notice that there are great similarities, at least for relatively high mass configurations, between the critical solutions and unstable boson stars in the ground state. (We do not perform studies involving boson stars with much lower masses, because of the dynamic range required for the spatial resolution of the finite difference code. Also, for a given numerical error tolerance, such low-mass critical solutions have much shorter lifetimes than larger-mass solutions and thus it is more difficult to obtain average properties of such short-lived critical solutions.) When we include nearly all of the complex-scalar mass in our comparisons, as shown in Figure $5(\mathrm{a})$, we see that the time-averaged properties of the critical solutions with lower masses, i.e. those further from the transition to instability, deviate from the curve of equilibrium configurations, and that the deviation increases as mass decreases. When we consider only the bulk of the boson star, however, we see very good agreement between the dynamically generated critical solutions and the unstable boson stars, computed from the static ansatz, as shown in Figure 5(b). The comparison between low-mass critical solutions and boson stars, shown in Figure [5, can be further illuminated by looking at a profile of the mass distribution as shown in Figure 6 .

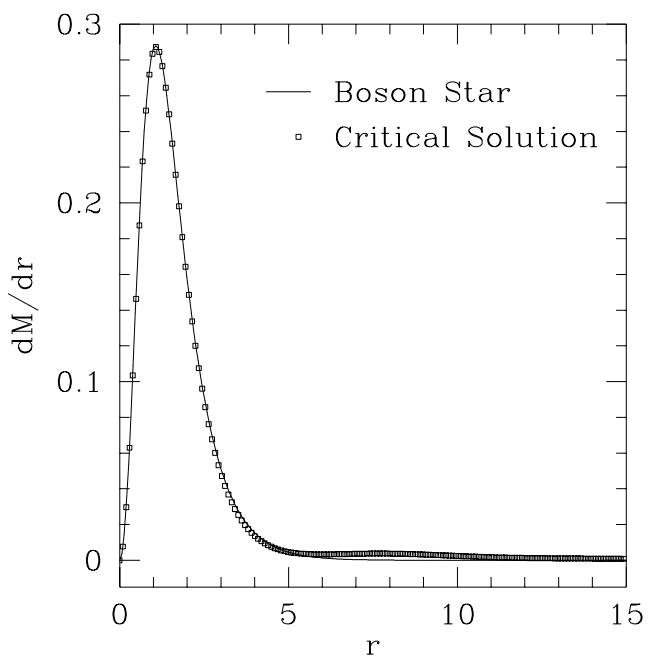

FIG. 6. Comparison of highly unstable (low-mass) critical solution and boson star. Squares show a critical solution resulting from a boson star having $\phi_{0}(0)=0.26 \times \sqrt{4 \pi}$. (The data has been reduced for graphing purposes; the actual spatial resolution in the simulation is four times finer than that shown in the figure.) The solid line shows a "best fit" (unstable) boson star we constructed by finding the time average of $|\phi(t, 0)|$ in the critical solution and using this as the value for $\phi_{0}(0)$ in the ODE integration routine which solves for the equilibrium (boson star) solutions. We see that there is a small halo near the outer edge of the solution $(r=8)$. The halo has the same relative magnitude when viewed in terms of the particle number distribution $\partial N / \partial r$. We discuss the halo phenomena further in Section V.

We see that there is a small halo near the outer edge of the solution $(r=8)$, and it is this which throws off our measurement of $R_{95}$ used for Figure . Despite the effect this has on the measurement of the radius $R_{95}$ of the star, we can still obtain a good fit of a boson star to the interior of the critical solution in the low-mass regime. We provide further discussion of these halos in Section V. in the critical solutions with higher total mass.

It is also worth noting that the critical solution best corresponds to a boson star in the "ground state," i.e., without any nodes in the distribution of the fields $\phi_{1}$ or $\phi_{2}$. Boson stars in excited states (i.e., having nodes in $\phi_{1}$ and $\phi_{2}$ ) have mass distributions which differ significantly from the critical solutions we obtain [20].

We wish to explain these simulation results in terms the quasi-normal modes of boson stars. Previous work in critical phenomena [1] - [8], 24] leads us to surmise that there is a single unstable mode present in the system which is excited when the boson star moves into the critical regime. The oscillatory behavior during the critical regime may be explainable in terms of the superposition of a stable oscillatory mode with the unstable mode. In the next section, we attempt to confirm these hypotheses by means of perturbation theory. 


\section{BOSON STAR STABILITY STUDY VIA LINEAR PERTURBATION THEORY}

We follow the work of Gleiser and Watkins [16]. For the perturbation calculations, we find it helpful to define the following metric functions:

$$
\begin{aligned}
e^{\nu(t, r)} & \equiv \alpha^{2} \\
e^{\lambda(t, r)} & \equiv a^{2} .
\end{aligned}
$$

and to rewrite the complex field $\phi(t, r)$ as

$$
\phi(t, r)=\left[\psi_{1}(t, r)+i \psi_{2}(t, r)\right] e^{-i \omega t},
$$

where $\psi_{1}$ and $\psi_{2}$ are real. (Note that this is a different decomposition of the field $\phi$ than (2.7), the one used in the previous sections.)

In these variables, the equilibrium quantities are

$$
\begin{aligned}
\lambda(t, r) & =\lambda_{0}(r) \\
\nu(t, r) & =\nu_{0}(r) \\
\psi_{1}(t, r) & =\phi_{0}(r) \\
\psi_{2}(t, r) & =0 .
\end{aligned}
$$

For the perturbation, we expand about the equilibrium quantities by first introducing four perturbation fields$\delta \lambda(t, r), \delta \nu(t, r), \delta \psi_{1}(t, r)$ and $\delta \psi_{2}(t, r)$-and then setting:

$$
\begin{aligned}
\lambda(t, r) & =\lambda_{0}(r)+\delta \lambda(t, r) \\
\nu(t, r) & =\nu_{0}(r)+\delta \nu(t, r) \\
\psi_{1}(t, r) & =\phi_{0}(r)\left(1+\delta \psi_{1}(t, r)\right) \\
\psi_{2}(t, r) & =\phi_{0}(r) \delta \psi_{2}(t, r) .
\end{aligned}
$$

These expressions are substituted into the relevant evolution and constraint equations (details in Appendix C), after which the resulting system can be reduced to the following system of two coupled second-order ordinary differential equations for $\delta \phi_{1}$ and $\delta \lambda$ :

$$
\begin{aligned}
\delta \psi_{1}^{\prime \prime}= & -\left(\frac{2}{r}+\frac{\nu_{0}^{\prime}-\lambda_{0}^{\prime}}{2}\right) \delta \psi_{1}^{\prime}-\frac{\delta \lambda^{\prime}}{r \phi_{0}^{2}}+e^{\lambda_{0}-\nu_{0}} \delta \ddot{\psi}_{1} \\
& -\left[\frac{\phi_{0}^{\prime}}{\phi_{0}}\left(\frac{\nu_{0}^{\prime}-\lambda_{0}^{\prime}}{2}+\frac{1}{r}\right)+\left(\frac{\phi_{0}^{\prime}}{\phi_{0}}\right)^{2}+\frac{1-r \lambda_{0}^{\prime}}{r^{2} \phi_{0}^{2}}+e^{\lambda_{0}-\nu_{0}} \omega^{2}-e^{\lambda_{0}}\right] \delta \lambda \\
& +2 e^{\lambda_{0}}\left[1+e^{-\nu_{0}} \omega^{2}+e^{-\lambda_{0}}\left(\frac{\phi_{0}^{\prime}}{\phi_{0}}\right)^{2}+r \phi_{0} \phi_{0}^{\prime}\right] \delta \psi_{1} \\
\delta \lambda^{\prime \prime}= & -\frac{3}{2}\left(\nu_{0}^{\prime}-\lambda_{0}^{\prime}\right) \delta \lambda^{\prime}+\left[4 \phi_{0}^{\prime 2}+\lambda_{0}^{\prime \prime}+\frac{2}{r^{2}}-\frac{\left(\nu_{0}^{\prime}-\lambda_{0}^{\prime}\right)^{2}}{2}-\frac{2 \nu_{0}^{\prime}+\lambda_{0}^{\prime}}{r}\right] \delta \lambda+e^{\lambda_{0}-\nu_{0}} \delta \ddot{\lambda} \\
& -4\left(2 \phi_{0} \phi_{0}^{\prime}-r e^{\lambda_{0}} \phi_{0}^{2}\right) \delta \psi_{1}^{\prime} \\
& -4\left[2 \phi_{0}^{\prime 2}-r e^{\lambda_{0}} \phi_{0}^{2}\left(2 \frac{\phi_{0}^{\prime}}{\phi_{0}}+\frac{2 \nu_{0}^{\prime}+\lambda_{0}^{\prime}}{2}\right)\right] \delta \psi_{1} .
\end{aligned}
$$

To perform the stability analysis (normal-mode analysis), we assume a harmonic time dependence, i.e.,

$$
\begin{gathered}
\delta \psi_{1}(t, r)=\delta \psi_{1}(r) e^{i \sigma t} \\
\delta \lambda(t, r)=\delta \lambda(r) e^{i \sigma t} .
\end{gathered}
$$

Note that (4.10) and (4.11) contain only second derivatives with respect to time, and because there are good reasons to assume $\sigma^{2}$ is purely real [1], 16], we only need to determine whether $\sigma^{2}$ is positive or negative to determine stability or instability, respectively.

Using the method described in Appendix C, we find the distribution for the squared frequency $\sigma_{0}^{2}$ of the fundamental mode, with respect to $\phi_{0}$, which is shown in Figure 7 . 
Superposed with the fundamental mode, we may have other modes at higher frequencies. Figure 8 shows the relation between first harmonic frequencies and $\phi_{0}(0)$.

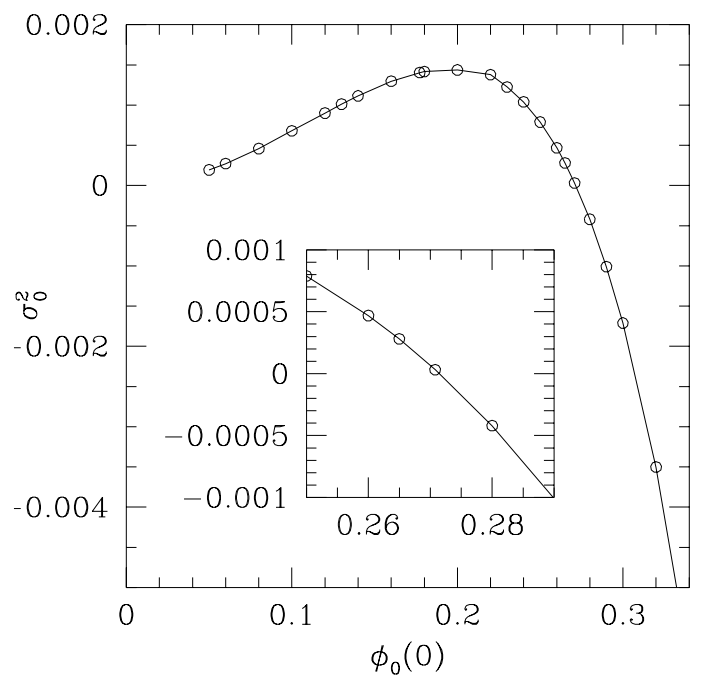

FIG. 7. Mode frequencies of boson stars: fundamental mode This plot shows a graph of $\sigma_{0}^{2}$, the squared frequency of the fundamental mode, versus the value of $\phi_{0}$ at the origin. Note that, as the inset shows, $\sigma_{0}^{2}$ crosses zero when $\phi_{0}(0) \simeq 0.27$, which corresponds to a boson star with the maximum possible mass. (The circles show actual values obtained, and the solid line simply connects these points.)

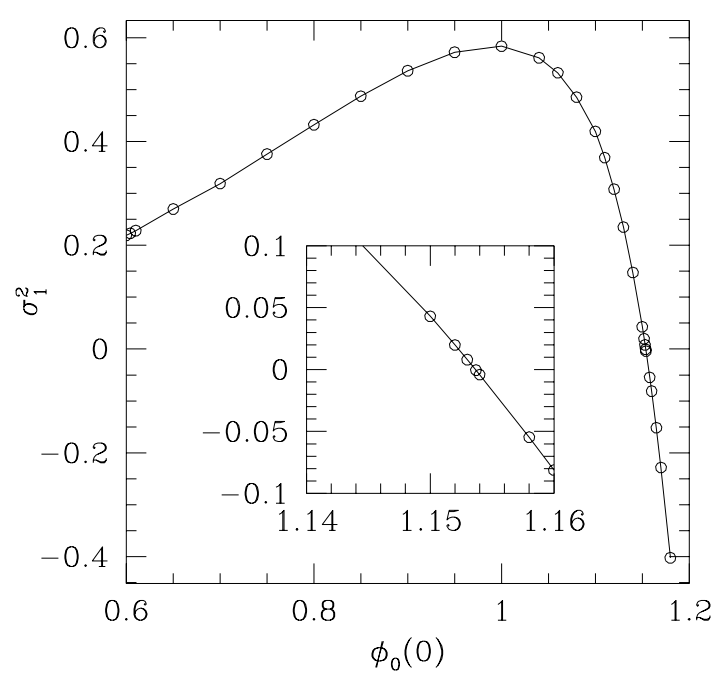

FIG. 8. Mode frequencies of boson stars: first harmonic mode This plot shows a graph of $\sigma_{1}^{2}$, the squared frequency of the first harmonic mode, versus the value of $\phi_{0}$ at the origin. Note that, as the inset shows, $\sigma_{1}^{2}$ crosses zero when $\phi_{0}(0) \simeq 1.15$, which corresponds to the first local minimum on the unstable branch of the mass vs. radius curve (see Figure 4). (The circles show actual values obtained, and the solid line simply connects these points.)

\section{COMPARISON OF PERTURBATION ANALYSIS AND SIMULATION DATA}

We wish to compare the results of our perturbation theory calculation with the oscillations of stable boson stars. Two differences exist between the conventions used in the perturbation theory calculation and those used in the boson star simulation data. The first difference is in the choice of the time coordinate. In the perturbation theory code, we choose a lapse of unity at the origin, whereas in the simulations we set the lapse to unity at spatial infinity. Thus we have the following mapping from the perturbation theory calculations to the simulations: 


$$
\left.\left.\sigma^{2}\right|_{\text {Perturbative }} \rightarrow \frac{\sigma^{2}}{\alpha^{2}}\right|_{\text {Simulation }}
$$

The other significant difference is in the way the complex field $\phi(t, r)$ is decomposed into constituent real fields. Thus we cannot directly compare $\phi_{1}$ and $\psi_{1}$, for example. We can, however, compare the modulus $|\phi|$ of the field. For the simulation data, the perturbation in $|\phi|$ can be taken directly from $\left(\phi_{1}^{2}+\phi_{2}^{2}\right)^{1 / 2}$. For the data obtained from perturbation theory, the perturbation in $|\phi|$ will be, to first order, $\phi_{0} \delta \psi_{1}$.

Before proceeding to the comparisons per se, we wish to point out that determining the unstable mode via numerical simulation of the full nonlinear system was very easy to do in comparison to the linear perturbation theory calculations.

\section{A. Unstable modes}

To measure the unstable mode, we again perform a series of simulations in which we allow a gaussian pulse from an addition real, massless Klein-Gordon field to impinge on a stable boson star.

By tuning the amplitude of this pulse (holding constant the width of the pulse and its initial distance from the boson star), we can generate a family of slightly different near-critical solutions depending on the amplitude of the initial gaussian pulse, and can tune down the initial magnitude of the unstable mode. By subtracting these slightly different near-critical solutions, we obtain a direct measurement of the unstable mode.

Considering a specific example, we start with a stable boson star which has an initial field value at the origin of $\phi_{0}(0)=0.04 \times \sqrt{4 \pi}$. By driving it with a gaussian pulse tuned to machine precision, we can cause this stable star to become a critical solution which persists for very long times, oscillating about a local equilibrium. The average value of $|\phi(t, 0)|$ is $\langle|\phi(t, 0)|\rangle \simeq 0.463$. We measure the unstable mode by subtracting data of a run which contained a gaussian pulse with an amplitude that differed by $10^{-14}$ from that of the pulse tuned to machine precision. We can then measure the growth factor of the unstable mode by taking the $L_{2}$ norm of this difference at various times, taking the logarithm, and fitting a straight line to it. From this, we obtain $\sigma \simeq 0.109 i$, or $\sigma^{2} \simeq-0.0118$. Because of the differences in time coordinate between the simulations and perturbation theory calculations, we need to compute $\sigma^{2} / \alpha^{2}$ in order to compare with the perturbation calculations. We find the average value of $1 / \alpha(t, 0)^{2}$ for the times listed above to be $\left\langle 1 / \alpha(t, 0)^{2}\right\rangle \simeq 3.80$, and thus we find $\sigma^{2} / \alpha^{2} \simeq-0.0450$. We choose to compare these perturbation theory results with data from a time in the simulation for which the difference in field values (for the two evolutions tuned slightly differently) is $\Delta|\phi(t, 0)| \simeq 8.4 \times 10^{-13}$. We use this value in the perturbation theory solver and arrive at $\sigma^{2} \simeq-0.045$, in good agreement with the value from the simulation. In Figures 9 and 10, we compare the graphs of the solutions for the unstable mode. In Figure 11 we show a comparison of the squared freqency values obtained from the linear perturbative analysis and those as measured in our simulations.
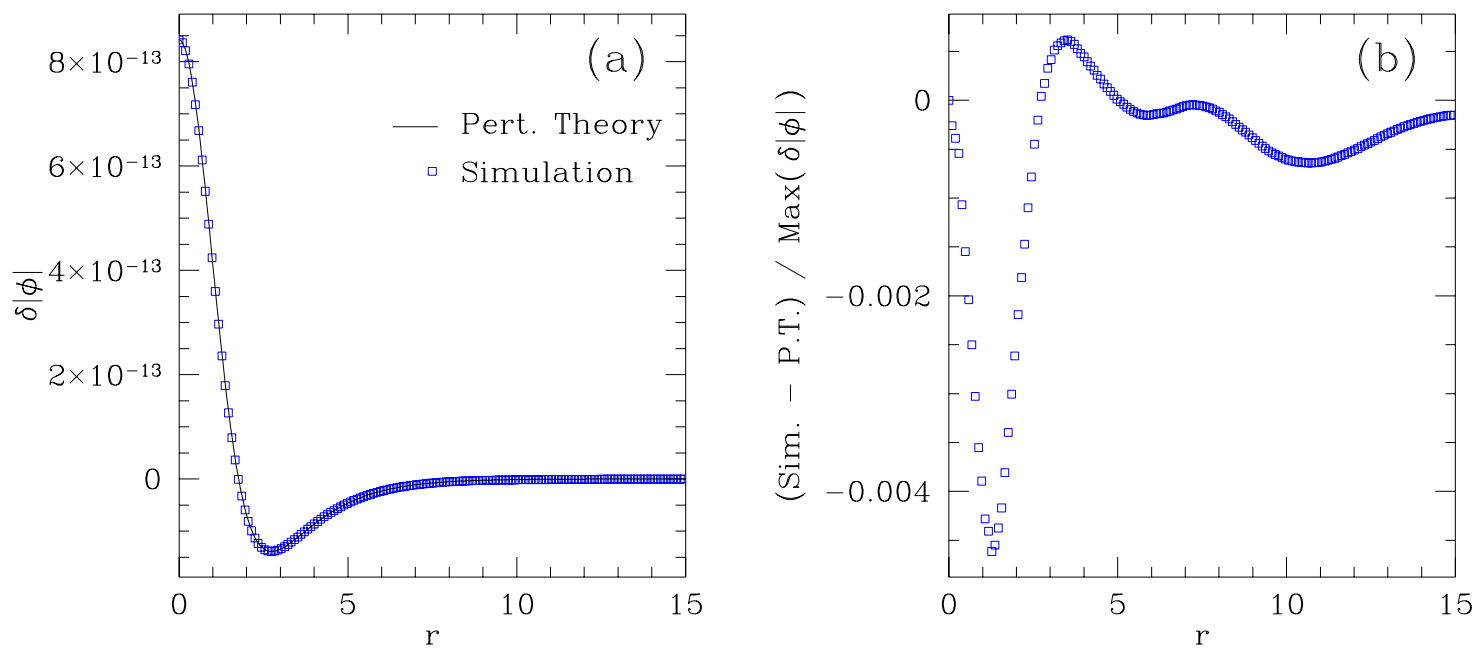

FIG. 9. Fundamental mode of unstable boson star. (a) The solid line shows $\phi_{0} \delta \psi_{1}$ from the perturbation theory calculations. The squares shows the difference between $|\phi|$ for two simulations for which the critical parameter $p$ differs by $10^{-14}$. (The data has been reduced for graphing purposes; the actual spatial resolution in the simulation is four times finer than what is shown in the figure.) Differences between the simulation data and perturbation theory results are below $1.1 \times 10^{-15}$. If a line were drawn connecting the squares, it would be indistinguishable, to the eye, from the perturbation theory line. Thus we provide a second graph (b) showing the difference of these results, where the scale is relative to the maximum value of $\delta|\phi|$. 

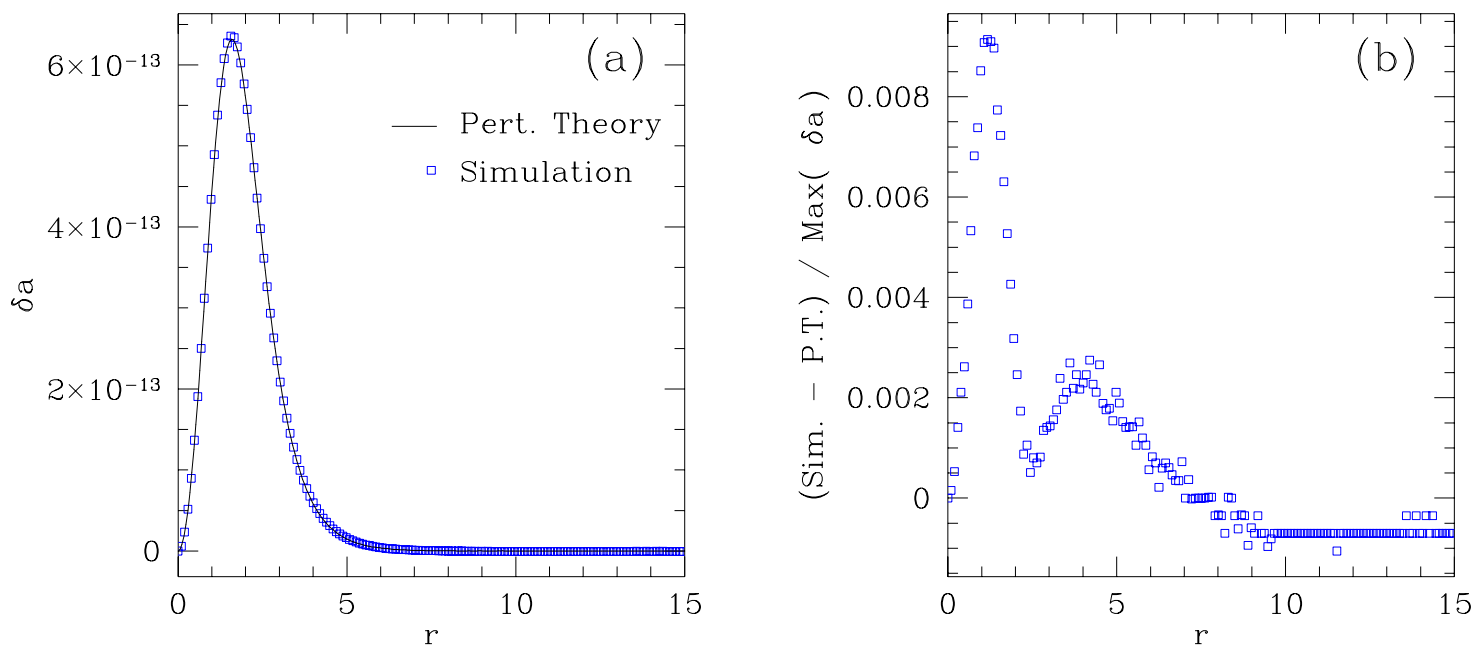

FIG. 10. Fundamental mode of unstable boson star. (a) The solid line shows the perturbation to the metric function $a$, as found from the perturbation theory calculations. The squares shows the difference between the metric function $a$ for two simulations for which the critical parameter $p$ differs by $10^{-14}$. (In the simulations, the spatial resolution was four times that shown in the figure.) (b) A plot of the difference between the mode obtained from the simulation and the mode obtained via perturbation theory, where the scale is relative to the maximum value of $\delta a$.

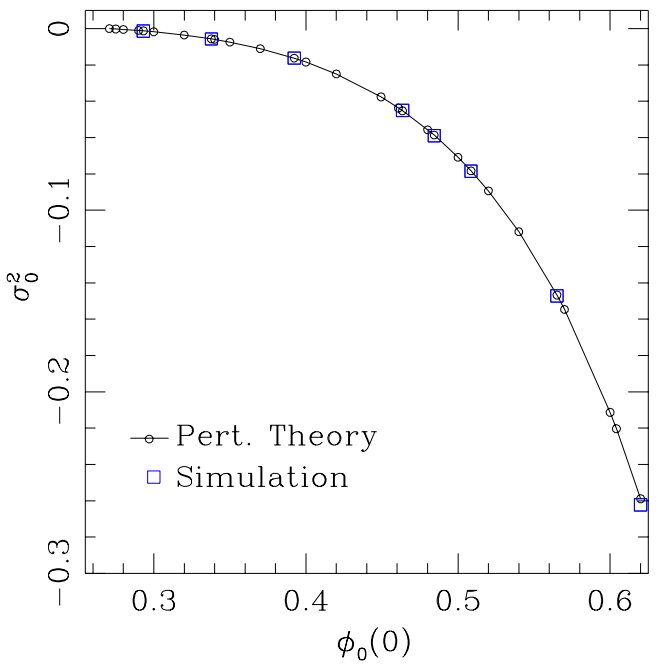

FIG. 11. Comparison of squared frequencies/Lyapunov exponents for unstable modes. The circles show a subset of the perturbation theory data as displayed in Figure 6 . The squares show the measurements obtained from our simulations. (The solid line simply connects the circles.) We note that the agreement between the two sets is good even for the more unstable, low-mass solutions. We also point out that the measurements of our simulations were performed along $r=0$, i.e.,, in the interior of the halo found in the low-mass solutions, which seems to strengthen the remarks at the end of section III, namely that, aside from the halo at the exterior of the critical solution, the critical solutions (of all masses) seem to correspond to unstable boson stars.

\section{B. Oscillatory modes}

We can also look at the oscillatory mode during the critical regime. We study the behavior of such a mode using the same technique we used to examine the fundamental mode of the unstable boson star: we subtract the data at one instant of time from the data at all other instants. Again, as a specific example, we use the same initial boson 
star as that used in the previous section. During the critical portion of the evolution, we notice an oscillation period of about $T \simeq 38.4$, and thus we obtain $\sigma=2 \pi / T \simeq 0.0261$. During this period, the average value of $1 / \alpha^{2}(t, 0)$ is about 3.80, and thus we find $\sigma^{2} / \alpha^{2} \simeq 0.102$. We take data from a moment in the middle of the oscillation period, and subtract it from the data at other times. We can then compare the perturbation theory results with simulation data at a local peak of the oscillation. For the local peak we chose at time $t=t_{p}$, the difference in modulus of the field was $\Delta\left|\phi\left(t_{p}, 0\right)\right| \simeq 0.0197$. Inserting this value into the perturbation theory code, we find $\sigma^{2} \simeq 0.102$ for this configuration. Thus we again find excellent agreement between the squared oscillation frequencies computed in perturbation theory and via simulation.

In Figures 12 and 13, we compare the functions obtained from the perturbation theory calculation with those from the simulation. We note that the agreement for the metric functions is very good for all radii, but the agreement in the fields begins to decline beyond $r=5$. Why do the graphs of $|\phi|$ not agree well for the first harmonic? This could be a consequence of our simplistic method of extracting this mode. While our method of simply subtracting different frames has worked well for our test cases of oscillations of stable boson stars, the first harmonic of the unstable star has a higher frequency and thus our graph could be subject to sampling effects. A better method would be to perform a Fourier transform in time for each grid point, and construct the higher harmonics in the field accordingly. There may be a simple resolution to the discrepancy in the graphs of $|\phi|,(2.7)$ and agreement in the graphs of the metric, our analysis does seem to indicate that the oscillations observed for this data in fact correspond to the first harmonic quasinormal mode of a boson star, however the analysis of the matter field needs further attention.

Finally, we must remark that we have been unable, using the fundamental and first harmonic modes of an unstable boson star, to construct a solution possessing a halo similar to that shown in Figure 6. We do not expect higher modes to be of any use here, because the halo is observed to oscillate with the same (single) frequency as the rest of the star. Since, as we described at the end of section III, the halo seems to be radiated away over time, we might not expect it to be described by the quasinormal modes (which conserve particle number) we have constructed.
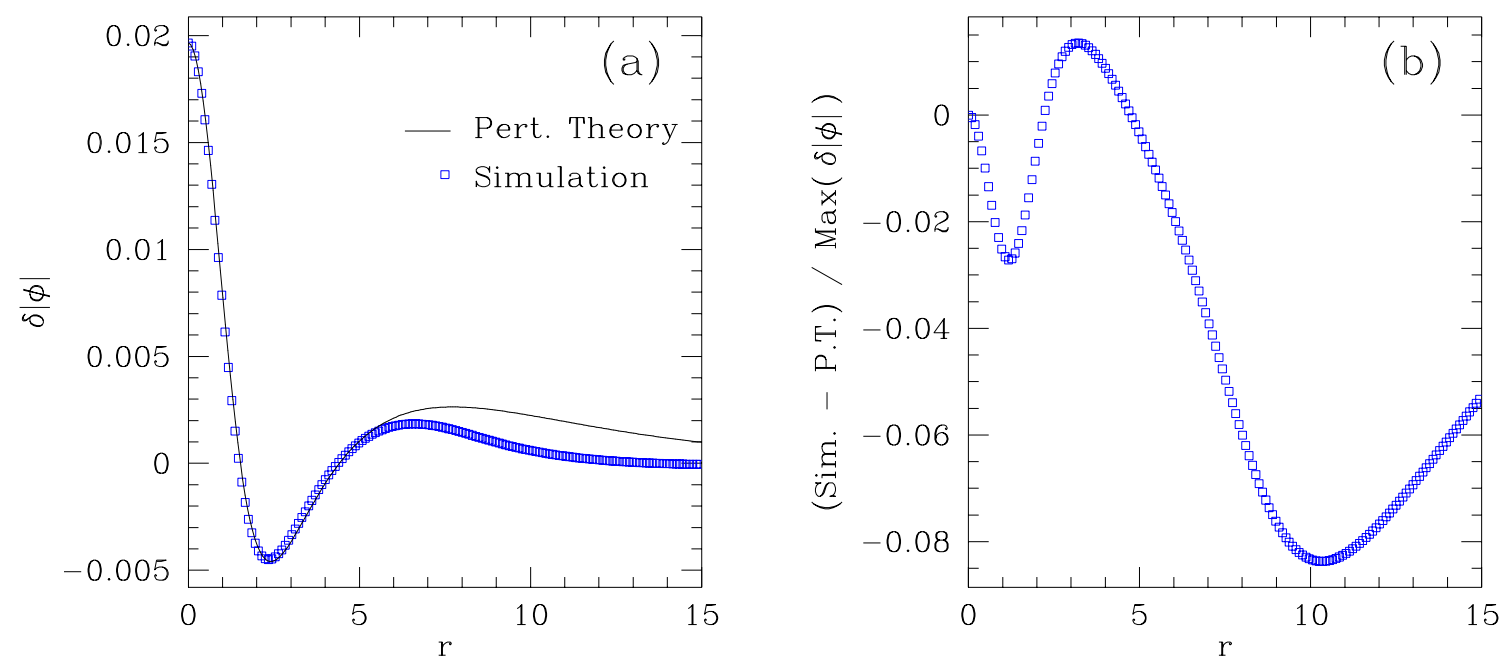

FIG. 12. First harmonic of an unstable boson star. (a) The solid line shows $\phi_{0} \delta \psi_{1}$ from the perturbation theory calculations. To obtain the squares, we took the simulation data and subtracted the Klein-Gordon field at one instant of time from the data at another instant. (The data in the simulations had a spatial resolution four times finer than what is shown in the figure.) (b) The squares show the difference between mode obtained from simulation and the mode obtained via perturbation theory, scaled relative to the maximum value of $\delta|\phi|$. As we describe in the text, the lack of agreement beyond $r \simeq 6$ may be an artifact of simplistic data analysis. The next figure shows that the metric quantities, which depend directly on the matter distribution (and thus on $|\phi|$ ), show a favorable comparison between the simulations and perturbation theory. 

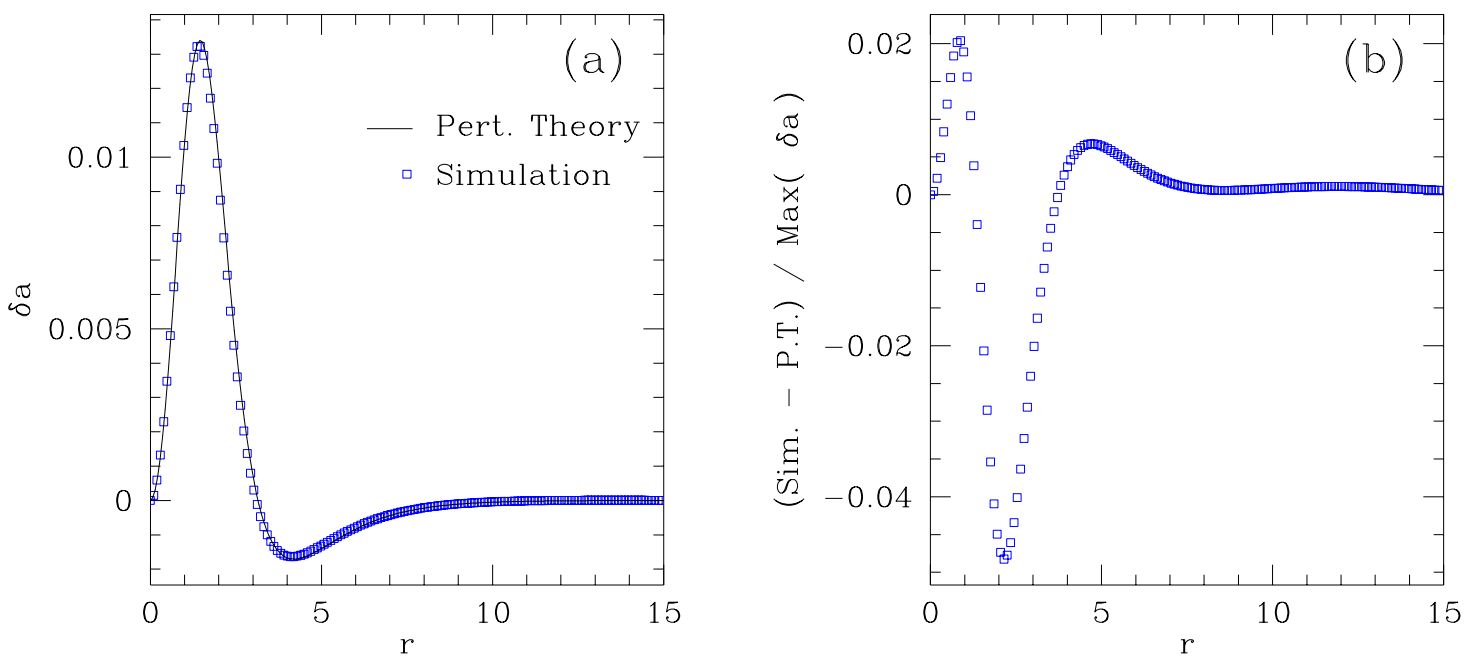

FIG. 13. First harmonic of an unstable boson star. (a) The solid line shows the perturbation to $a$ as found from perturbative calculations. To plot the squares, we took the simulation data and subtracted the metric function $a$ at one instant of time from the data at another instant. (The spatial resolution in the simulation was four times finer than what is shown in the figure.) (b) The squares show the difference between the simulation data and the results of linear perturbation theory, scaled relative to the maximum value of $\delta a$. The close fit between these results indicates that the oscillations observed in the critical solutions correspond to stable oscillatory modes in an unstable boson star.

\section{HALOS}

We have strong evidence that that the critical solutions correspond to unstable boson stars, but the principal point of disagreement is existence of a "halo" of massive field which resides in the "tail" of the solution. It is our contention that this halo is not part of the true critical solution, but rather, is an artifact of the collision with the massless field.

In particular, the halo seems to be a remnant of the original (stable) boson star which is not induced to collapse with the rest of the star to form the true critical solution. We find that such a halo is observable in nearly all but the most massive (least unstable) critical solutions we have considered, and that its size tends to increase as less massive (more unstable) solutions are generated. The fact that the halo thus decreases as we approach the turning point only makes sense - a stable boson star very close to the turning point needs very little in the way of a perturbation from the massless field to be "popped" over to the unstable branch, and the final, unstable configuration, will, of course, be very close to the initial state.

Additionally, we note that in all cases we have exmained, the field comprising the halo oscillates with nearly the same (single) frequency as the rest of the solution. This indicates that the halo is not explainable in terms of additional higher-frequency modes.

As one might expect, the properties of the halo are not universal, i.e. they are quite dependent on the type of initial data used. In contrast, the critical solution interior to the halo is largely independent of the form of the initial data. To demonstrate this, we use two families of initial data, given by a "gaussian" of Family I in Table li and a "kink" of Family II. A series of snapshots from one such pair of evolutions is shown in Figure 14. We find different amounts of mass transferred from the massless to the massive field for the kink and gaussian data, as shown in Figure 15, yet the central values of the field oscillate about nearly the same value at nearly the same frequency. Both calculations start with identical boson stars with $|\phi(0,0)|=0.04 \times \sqrt{4 \pi}$. In the critical regimes, this becomes $\langle|\phi(t, 0)|\rangle=0.130 \times \sqrt{4 \pi}$ for the solution obtained from the gaussian data, and $\langle|\phi(t, 0)|\rangle=0.135 \times \sqrt{4 \pi}$ for the kink data. As already noted, the oscillation periods are also quite similar, differing by about $3 \%$, and the massess interior to the halo are also quite comparable. In particular, it seems quite remarkable that the differences in mass interior to the halo for the two families are much smaller than the mass transferred from the real field in either case. 


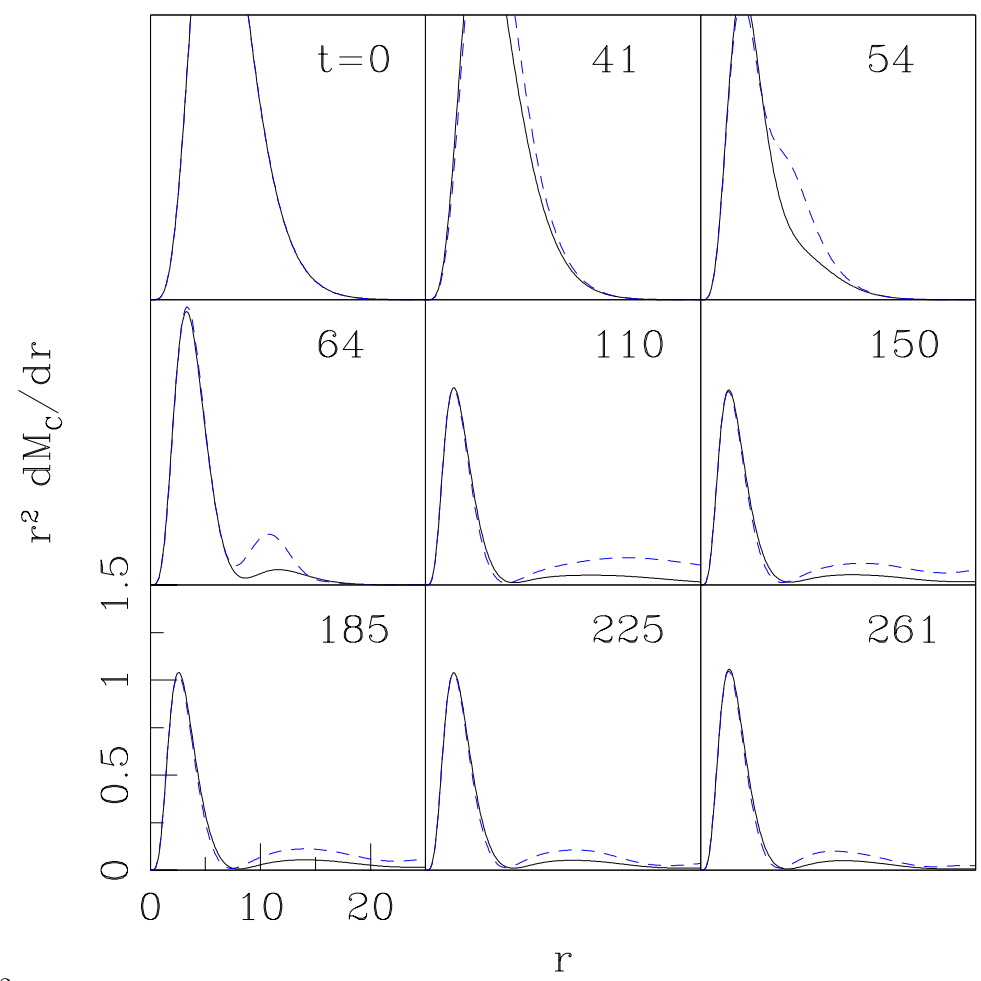

FIG. 14. Evolution of $r^{2} d M_{C} / d r$ for for two different sets of initial data. Both sets contain the same initial boson star, but the massless field $\phi_{3}$ for one set is given by a "gaussian" of Family I (solid line) with $r_{0}=30$, and $\Delta=8$ whereas for the other set $\phi_{3}$ is given by a "kink" of Family II (dashed line) with $r_{0}=35$ and $\Delta=3$. The variable $A$ is varied (independently for each family) as the parameter $p$ to obtain the critical solution. (Note that after $t \simeq 60$, the massless field has completely left the domain shown in the figure.) We have multiplied $d M_{C} / d r$ by $r^{2}$ to highlight the dynamics of the halo; thus the main body of the solution appears to decrease in size as it moves to lower values of $r$. The kink data produces a larger and much more dynamical halo, but interior to the halo, the two critical solutions match closely — and also match the profile of an unstable boson star. Thus, the portion of the solution which is "universal" corresponds to an unstable boson star.

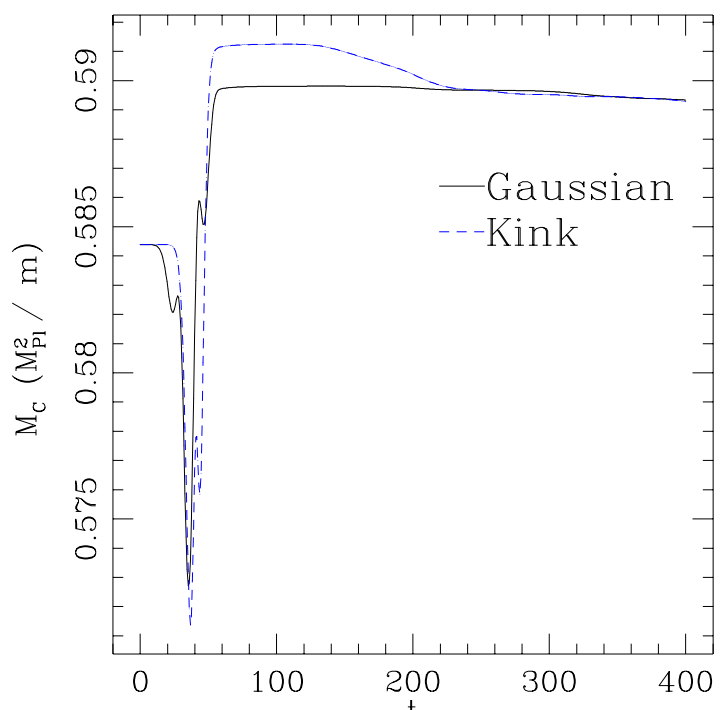

FIG. 15. $M_{C}$ vs. time for the two evolutions shown in Figure 14. Mass transfer from the real to the complex field occurs from $t \simeq 30$ to $t \simeq 60$, i.e. while the supports of the fields overlap. There is more mass transferred using the kink data, and yet the mass falls off rapidly. The mass of the kink data acquires a value very close to the mass of the gaussian data, which is itself decreasing slowly with time. We see that, beyond $t \simeq 250$, the difference in mass between the two solutions is very small compared with the amount of mass transferred from the real field. 
If we consider the inner edge of the halo to be where $\partial|\phi| / \partial r=0$ at some finite radius (e.g., $r \simeq 5$ in Figure 6), and look at the data between $r=0$ and the inner edge of the halo, we find good agreement between this data and the profile of a boson star. This can be seen in both Figures 6 and 16 .

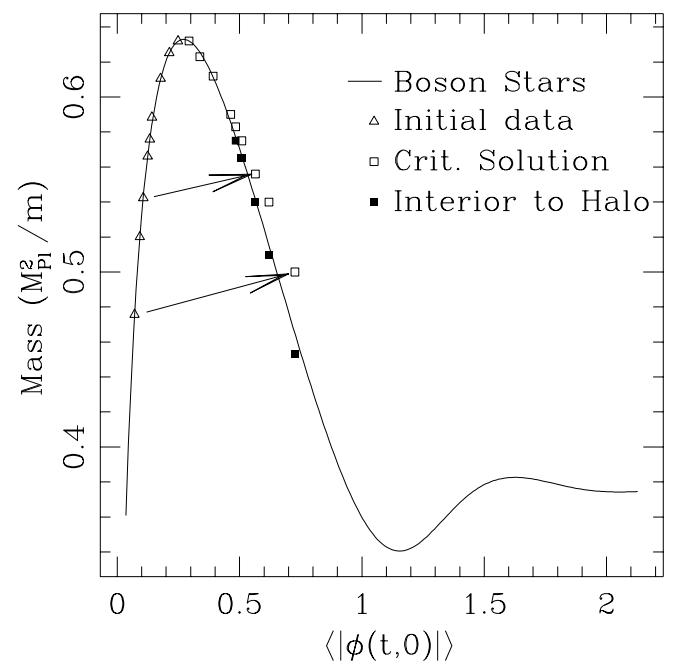

FIG. 16. Mass vs. $\langle|\phi(t, 0)|\rangle$, the time average of the central value of the field for equilibrium configurations of boson stars (solid line), initial data (triangles) and critical solutions (open and filled squares). Arrows are given to help match initial data with the corresponding critical solution. Points on the solid line to the left of the maximum mass $M_{\max } \simeq 0.633 M_{P l}^{2} / m$ correspond to stable boson stars, whereas those to the right of the maximum correspond to unstable stars. The data is the same as that used for Figure , with data from one further evolution added at the bottom of the mass range. The open squares show the time average of the mass and $|\phi(t, 0)|$ of some critical solutions, and the filled squares show the same quantities evaluated between $r=0$ and the inner edge of the halo, defined to be the point where $\partial|\phi| / \partial r=0$ for finite $r$. The mass of the critical solution is in general greater than the mass of the initial data, however the mass inside the halo of the critical solution is less than the mass of the initial data.

We suspect that the halo is radiated over time (via scalar radiation, or "gravitational cooling" [23]) for all critical solutions. We find, however, that the time scale for radiation of the halo is comparable to the time scale for dispersal or black hole formation for each (nearly) critical solution we consider. Thus, while we see trends which indicate that the halo is indeed radiating, we are not able to demonstrate this conclusively for a variety of scenarios. With higher numerical precision, one might be able to more finely tune out the unstable mode, allowing more time to observe the behavior of the halo before dispersal or black hole formation occur.

\section{CONCLUSIONS}

We have shown that it is possible to induce gravitational collapse and, in particular, Type I critical phenomena in spherically-symmetric boson stars in the ground state, by means of "perturbations" resulting from gravitational interaction with an in-going pulse from a massless real scalar field. Through this interaction, energy is transferred from the real to the complex field, and complex field is "driven" and "squeezed" to form a critical solution. The massless field is not directly involved in the critical behavior observed in the complex massive field; the critical solution itself appears to correspond to a boson star, which, at any finite distance from criticality in parameter space, exhibits a superposition of stable and unstable modes.

Specifically, for initial data consisting of a boson star with nearly the maximum possible mass of $M_{\max } \simeq$ $0.633 M_{p l}^{2} / m$, the resulting critical solution oscillates about a state which has all the features of the corresponding unstable boson star in the ground state, having the same mass as the initial star. This result is reminiscent of the study by Brady et al. [8], who found that the Type I critical solutions for a real massive scalar field corresponded to the oscillating soliton stars of Seidel and Suen [18. For boson stars with a mass somewhat less than $M_{\max }$, e.g., $0.9 M_{\max }$ or less, however, we find less than complete agreement between the critical solution and an unstable boson star of comparable mass. This is evidenced by the presence of an additional spherical shell or "halo" of matter in the critical solution, located in what would be the tail of the corresponding boson star. Interior to this halo, we find that 
the critical solution compares favorably with the profile of an unstable boson star. Additionally, we have shown that the halo details depend on the specifics of the perturbing massless field, and we conjecture that, in the infinite time limit, the halo would be radiated away.

In order to extend the comparison between the critical solutions and boson stars, we have verified and applied the linear perturbation analysis presented by Gleiser and Watkins [16], extending their work by providing an algorithm to obtain modes with nonzero frequency. We have used this algorithm to give quantitative distributions of mode frequency vs. central density of the boson star for the first two modes, as well as to solve for the modes to compare with our simulation results. We have found that the unstable mode in the critical solutions have the same growth rate as the unstable mode of boson stars, and that the mode shapes also compare quite favorably. We noted that the unstable mode of these boson stars was determined much more easily by solving the full nonlinear set of evolution equations, rather than via linear perturbation theory. The oscillations observed in the critical solution also indicated agreement with first harmonic mode obtained via perturbation theory, however the oscillatory mode in $|\phi|$ showed poor agreement at large radii, and awaits more careful analysis.

Future work may include simulations of the critical solutions of low mass using higher numerical precision to further tune away the initial amplitude of the unstable mode, thus allowing more time to observe the the small halo (i.e., whether it is in fact being radiated away). We would also hope to obtain better agreement between simulation and perturbation theory for the first harmonic mode of the field $|\phi|$, perhaps using a more sophisticated method of extracting modes from the simulation. Another direction worthy of note would be to begin the simulation with a pulse of the complex field (instead of specifically a boson star) tune the height of the pulse to find the critical solutions via interpolation, and then compare the resulting critical solutions with our results obtained by perturbing boson stars.

Finally, we find it worthwhile to investigate similar scenarios for neutron stars. While there have been studies regarding the explosion of neutron stars near the minimum mass (e.g., [33], [34]), we would like to see whether neutron stars of non-minimal mass can be driven to explode via dispersal from a critical solution. This may take the form of a neutron star approaching the onset of instability via slow accretion, or by being driven across the stability graph via violent heating from some other matter source, in a manner similar to the perturbations of boson stars we have considered in this paper.

\section{ACKNOWLEDGMENTS}

This research was supported by NSERC and by NSF PHY9722068 Some computations were carried out on the vn.physics.ubc. ca Beowulf cluster which was funded by the Canadian Foundation for Innovation. Other calculations were performed using the Cray T3E at the Texas Advanced Computing Center.

\section{APPENDIX A: BOSON STAR MODE FREQUENCIES}

In this Appendix we have tabulated some sample values from the perturbation theory calculations. The values and uncertainties expressed in the table captions were determined by integrating (4.10) and (4.11) to various maximum radii, for a range of error tolerances in the integration routines. The values and uncertainties given in the tables were chosen to express the variation in our results. 
TABLE II. Shooting Parameters: Fundamental Mode. The values of $\phi_{0}(0)$ are exact. Other quantities are given within an uncertainty of \pm 1 in the last significant digit.

\begin{tabular}{cccr}
\hline \hline$\phi_{0}(0)$ & $\omega$ & $\delta \lambda^{\prime \prime}(0) / \delta \psi_{1}(0)$ & $\sigma^{2}$ \\
\hline $6.0 \mathrm{E}-02$ & $1.0417 \mathrm{E}+00$ & $1.68 \mathrm{E}-01$ & $0.28 \mathrm{E}-03$ \\
$1.0 \mathrm{E}-01$ & $1.0727 \mathrm{E}+00$ & $0.29 \mathrm{E}+00$ & $0.67 \mathrm{E}-03$ \\
$1.4 \mathrm{E}-01$ & $1.1067 \mathrm{E}+00$ & $0.43 \mathrm{E}+00$ & $1.11 \mathrm{E}-03$ \\
$1.8 \mathrm{E}-01$ & $1.1440 \mathrm{E}+00$ & $0.59 \mathrm{E}+00$ & $1.41 \mathrm{E}-03$ \\
$2.2 \mathrm{E}-01$ & $1.1849 \mathrm{E}+00$ & $0.77 \mathrm{E}+00$ & $1.31 \mathrm{E}-03$ \\
$2.6 \mathrm{E}-01$ & $1.2299 \mathrm{E}+00$ & $0.98 \mathrm{E}+00$ & $0.45 \mathrm{E}-03$ \\
$2.7 \mathrm{E}-01$ & $1.2419 \mathrm{E}+00$ & $1.04 \mathrm{E}+00$ & $0.05 \mathrm{E}-03$ \\
$2.8 \mathrm{E}-01$ & $1.2542 \mathrm{E}+00$ & $1.10 \mathrm{E}+00$ & $-0.43 \mathrm{E}-03$ \\
$3.0 \mathrm{E}-01$ & $1.2796 \mathrm{E}+00$ & $1.24 \mathrm{E}+00$ & $-1.71 \mathrm{E}-03$ \\
$4.0 \mathrm{E}-01$ & $1.4281 \mathrm{E}+00$ & $2.08 \mathrm{E}+00$ & $-1.84 \mathrm{E}-02$ \\
$5.0 \mathrm{E}-01$ & $1.6215 \mathrm{E}+00$ & $3.45 \mathrm{E}+00$ & $-7.09 \mathrm{E}-02$ \\
$6.0 \mathrm{E}-01$ & $1.8777 \mathrm{E}+00$ & $5.79 \mathrm{E}+00$ & $-2.11 \mathrm{E}-01$ \\
\hline \hline
\end{tabular}

TABLE III. Shooting Parameters: First Harmonic Mode. The values of $\phi_{0}(0)$ are exact, $\omega$ is given within an uncertainty of \pm 1 in the last significant digit, and the other quantities are given within an uncertainty of \pm 2 in the last significant digit.

\begin{tabular}{cccr}
\hline \hline$\phi_{0}(0)$ & $\omega$ & $\delta \lambda^{\prime \prime}(0) / \delta \psi_{1}(0)$ & $\sigma^{2}$ \\
\hline $6.00 \mathrm{E}-01$ & $1.8777 \mathrm{E}+00$ & $0.63 \mathrm{E}+01$ & $0.22 \mathrm{E}+00$ \\
$7.00 \mathrm{E}-01$ & $2.2230 \mathrm{E}+00$ & $1.13 \mathrm{E}+01$ & $0.32 \mathrm{E}+00$ \\
$8.00 \mathrm{E}-01$ & $2.6963 \mathrm{E}+00$ & $2.09 \mathrm{E}+01$ & $0.43 \mathrm{E}+00$ \\
$9.00 \mathrm{E}-01$ & $3.3536 \mathrm{E}+00$ & $4.11 \mathrm{E}+01$ & $0.53 \mathrm{E}+00$ \\
$1.00 \mathrm{E}+00$ & $4.2714 \mathrm{E}+00$ & $0.84 \mathrm{E}+02$ & $0.54 \mathrm{E}+00$ \\
$1.10 \mathrm{E}+00$ & $5.5471 \mathrm{E}+00$ & $1.77 \mathrm{E}+02$ & $0.42 \mathrm{E}+00$ \\
$1.12 \mathrm{E}+00$ & $5.8555 \mathrm{E}+00$ & $2.07 \mathrm{E}+02$ & $3.05 \mathrm{E}-01$ \\
$1.14 \mathrm{E}+00$ & $6.1842 \mathrm{E}+00$ & $2.41 \mathrm{E}+02$ & $1.46 \mathrm{E}-01$ \\
$1.15 \mathrm{E}+00$ & $6.3566 \mathrm{E}+00$ & $2.59 \mathrm{E}+02$ & $4.30 \mathrm{E}-02$ \\
$1.16 \mathrm{E}+00$ & $6.5346 \mathrm{E}+00$ & $2.80 \mathrm{E}+02$ & $-8.11 \mathrm{E}-02$ \\
$1.17 \mathrm{E}+00$ & $6.7184 \mathrm{E}+00$ & $3.02 \mathrm{E}+02$ & $-2.28 \mathrm{E}-01$ \\
$1.18 \mathrm{E}+00$ & $6.9083 \mathrm{E}+00$ & $3.26 \mathrm{E}+02$ & $-4.01 \mathrm{E}-01$ \\
\hline \hline
\end{tabular}

\section{APPENDIX B: FINITE DIFFERENCE ALGORITHM}

We approximate the continuum field quantities $\left\{\alpha, a, \Pi_{1}, \Pi_{2}, \Pi_{3}, \Phi_{1}, \Phi_{2}, \Phi_{3}, \phi_{1}, \phi_{2}, \phi_{3}\right\}$ by a set of grid functions, quantities which are obtained via the solution of finite difference approximations to the partial differential equations (2.8), (2.11) - (2.14) on a domain which has been discretized into a regular mesh (i.e. lattice) with mesh spacing $\Delta r$ in space and $\Delta t$ in time. For a grid function $u$, we denote the value of the grid function in the mesh location $j$ in space and $n$ in time by $u_{j}^{n}$, e.g,

$$
\alpha_{j}^{n} \simeq \alpha(n \Delta t,(j-1) \Delta r),
$$

where $\alpha(n \Delta t,(j-1) \Delta r)$ is the corresponding value for the continuum solution.

The initial data is obtained via "shooting," a standard method of solving ordinary differential equations, in a way essentially the same as that found in [12]. The numerical method used for evolving the system of equations is a leapfrog scheme, which is an explicit scheme requiring data at two previous time steps, $n$ and $n-1$, to compute a value at the next time step $n+1$. Given a discretization of scale of order $h$ in time and space, the leapfrog scheme is $\mathcal{O}\left(h^{2}\right)$ accurate. Throughout the mesh, the ratio $\lambda_{\mathrm{CFL}} \equiv \Delta t / \Delta r$ is kept at a constant value, which must be less than unity due to the stability requirements of the leapfrog scheme.

To aid in the presentation of the difference equations, we define the following operators [26]: 


$$
\begin{gathered}
\Delta_{0}^{t} u_{j}^{n}=\frac{u_{j}^{n+1}-u_{j}^{n-1}}{2 \Delta t} \\
\Delta_{0}^{r} u_{j}^{n}=\frac{u_{j+1}^{n}-u_{j-1}^{n}}{2 \Delta r} \\
\Delta_{+}^{r} u_{j}^{n}=\frac{u_{j+1}^{n}-u_{j}^{n}}{\Delta r} \\
\Delta_{3}^{r} u_{j}^{n}=3 \frac{u_{j+1}^{n}-u_{j-1}^{n}}{\left(r_{j+1}\right)^{3}-\left(r_{j-1}\right)^{3}} .
\end{gathered}
$$

We also define the averaging operator

$$
\mu_{+}^{r} u_{j}^{n}=\frac{1}{2}\left(u_{j+1}^{n}+u_{j}^{n}\right)
$$

which takes precedence over other algebraic operations, e.g.

$$
\mu_{+}^{r}\left(\frac{f g^{2}}{h}\right)=\frac{\mu_{+}^{r} f_{j}^{n}\left(\mu_{+}^{r} g_{j}^{n}\right)^{2}}{\mu_{+}^{r} h_{j}^{n}} .
$$

The evolution equations, which are applied to each field $\left\{\Phi_{i}, \Pi_{i}, i=1,2,3\right\}$ can then be written as:

$$
\begin{gathered}
\Delta_{0}^{t} \Phi_{j}^{n}=\Delta_{0}^{r}\left(\frac{\alpha}{a} \Pi\right)_{j}^{n} \\
\Delta_{0}^{t} \Pi_{j}^{n}=\Delta_{3}^{r}\left(\frac{r^{2} \alpha}{a} \Phi\right)_{j}^{n}-2(\alpha a \phi)_{j}^{n}
\end{gathered}
$$

where the last term in the evolution equation for $\Pi$ is not applied to the massless field.

Our boundary conditions are as follows: First, by regularity at the origin, we have

$$
\Phi_{1}^{n}=0
$$

for all $n$. To obtain $\Pi_{1}^{n+1}$ we employ a "quadratic fit" at the advanced time,

$$
\Pi_{1}^{n+1}=\frac{4 \Pi_{2}^{n+1}-\Pi_{3}^{n+1}}{3},
$$

which is based on the regularity condition, $\lim _{r \rightarrow 0} \Pi(t, r)=\Pi_{0}(t)+r^{2} \Pi_{2}(t)+\cdots$.

A significant challenge in the numerical solution of these equations is the problem of the outer boundary condition for the massive field. Numerous authors have proposed methods to handle this. Having tried various methods including first order expansions of the dispersion relation [18, sponge filters [27], and operator splitting [28], we were unable to obtain a scheme which produced results superior to the simple Sommerfeld condition one uses for massless fields [29]. Since, however, the Sommerfeld condition is still inadequate for massive fields, we have chosen to run our simulations on a grid large enough that the outer boundary is out of causal contact with the region of interest for the time the simulation runs. So, for example, if we are interested in a region $0 \leq r \leq 50$ and times $0 \leq t \leq 400$, then we place the outer boundary $r_{J} \geq 450$. (While unbounded phase velocities are a feature of the Klein-Gordon equation, we can argue on physical grounds as well as see quite clearly in simulations that it is the group velocity 
which is the important quantity in the numerical evolutions, and this is sub-luminal.) Recent work using a shifted coordinate system, with a shift vector that is vanishing in some region near $r=0$ but increases to unity as $r \rightarrow r_{J}$, shows promise as a means of handling the challenge of the boundary condition for the massive field [30], and this method may be employed in future work. Thus the outer boundary condition we employ is [31]:

$$
\Phi_{J}^{n+1}=\left(\frac{3}{\Delta t}+\frac{3}{\Delta r}+\frac{2}{r_{J}}\right)^{-1}\left(\frac{4 \Phi_{J}^{n}-\Phi_{J}^{n-1}}{\Delta t}+\frac{4 \Phi_{J-1}^{n+1}-\Phi_{J-2}^{n+1}}{\Delta r}\right)
$$

and an analagous equation is used for each $\Pi$ variable.

After these evolved variables are obtained at the $n+1$ time step, we apply a form of numerical dissipation advocated by Kreiss and Oliger [25]. This is applied to both $\Phi_{j}^{n+1}$ and $\Pi_{j}^{n+1}$ in the same manner. So, for instance we set

$$
\Phi_{j}^{n+1}:=\Phi_{j}^{n+1}-\frac{\epsilon}{16}\left(\Phi_{j+2}^{n-1}-4 \Phi_{j+1}^{n-1}+6 \Phi_{j}^{n-1}-4 \Phi_{j-1}^{n-1}+\Phi_{j-2}^{n-1}\right),
$$

where $\epsilon(0<\epsilon<1)$ is an adjustable parameter: typically, we use $\epsilon=0.5$.

The preceeding equations describe the "evolution" aspect of the code. The other variables are evolved in a "constrained" manner, i.e. they are obtained on the spacelike hypersurface $n+1$ after the fields $\Phi_{j}^{n+1}$ and $\Pi_{j}^{n+1}$ have been calculated. The field values $\phi_{j}^{n+1}$ are obtained by updating the value at the outer boundary $j=J$ according to

$$
\Delta_{0}^{t} \phi_{J}^{n}=+\left(\frac{\alpha}{a} \Pi\right)_{j}^{n}
$$

and then integrating inward from $j=J$ to $j=1$ along the spatial hypersurface at $n+1$ :

$$
\Delta_{+}^{r} \phi_{j}=\mu_{+}^{r} \Phi_{j} .
$$

The Hamiltonian constraint (2.11) can be solved at each time step once all the field variables have been computed for the advanced time step. We use the variable $A \equiv \ln a$ to avoid loss of precision near the origin in the following finite difference approximation, which is evaluated at the advanced time step $n+1$ :

$$
\Delta_{+}^{r} A_{j}=\mu_{+}^{r}\left(\frac{1-e^{A}}{2 r}+\frac{r}{2}\left[\Pi_{1}^{2}+\Pi_{2}^{2}+\Pi_{3}^{2}+\Phi_{1}^{2}+\Phi_{2}^{2}+\Phi_{3}^{2}+e^{A}\left(\phi_{1}^{2}+\phi_{2}^{2}\right)\right]\right)_{j} .
$$

This equation is solved using a pointwise Newton iteration, i.e. given a value of $A_{j}^{n+1}$ (such as $A_{1}^{n+1}=0$ at the origin), we find the next value $A_{j+1}^{n+1}$ outward along the spatial hypersurface by solving (B8) via Newton's method.

The slicing condition can be solved once the field variables and the metric function $a$ have been obtained at the advanced time step, using the following linear algebraic relation:

$$
\alpha_{j+1}^{n+1}=\alpha_{j}^{n+1} \cdot \frac{(1 / \Delta r)+Z}{(1 / \Delta r)-Z}
$$

where

$$
Z \equiv \mu_{+}^{r}\left(\frac{a^{2}-1}{2 r}\right)_{j}+\frac{\Delta_{+}^{r} a_{j}}{\mu_{+}^{r} a_{j}}-\mu_{+}^{r}\left[r a^{2} m^{2}\left(\phi_{1}^{2}+\phi_{2}^{2}\right)\right]_{j} .
$$

\section{APPENDIX C: DETAILS OF LINEAR STABILITY ANALYSIS}

Following Gleiser and Watkins [16], we write the most general time-dependent, spherically-symmetric metric as

$$
d s^{2}=-e^{\nu(t, r)} d t^{2}+e^{\lambda(t, r)} d r^{2}+r^{2} d \Omega,
$$

and decompose the complex massive field $\phi(t, r)$ via 


$$
\phi(t, r)=\left[\psi_{1}(t, r)+i \psi_{2}(t, r)\right] e^{-i \omega t},
$$

where $\psi_{1}$ and $\psi_{2}$ are real.

In these variables, the Hamiltonian constraint and slicing condition can be written as

$$
\begin{gathered}
\lambda^{\prime}=\frac{1-e^{\lambda}}{r}+r\left(e^{\lambda-\nu}\left[\left(\dot{\phi}_{1}+\omega \psi_{2}\right)^{2}+\left(\dot{\phi}_{2}-\omega \psi_{1}\right)^{2}\right]+\psi_{1}^{\prime 2}+\psi_{2}^{\prime 2}+e^{\lambda}\left(\psi_{1}^{2}+\psi_{2}^{2}\right)\right) \\
\nu^{\prime}=\lambda^{\prime}+2 \frac{e^{\lambda}-1}{r}-2 r e^{\lambda}\left(\psi_{1}^{2}+\psi_{2}^{2}\right)
\end{gathered}
$$

where a prime $\left({ }^{\prime}\right)$ denotes $\partial / \partial r$ and an overdot $\left({ }^{\circ}\right)$ denotes $\partial / \partial t$.

The Klein Gordon equation yields:

$$
\psi_{1}^{\prime \prime}+\left(\frac{2}{r}+\frac{\nu^{\prime}-\lambda^{\prime}}{2}\right) \psi_{1}^{\prime}+e^{\lambda}\left(e^{-\nu} \omega^{2}-1\right) \psi_{1}-e^{\lambda-\nu} \ddot{\psi}_{1}+e^{\lambda-\nu} \frac{\dot{\nu}-\dot{\lambda}}{2}\left(\dot{\psi}_{1}+\omega \psi_{2}\right)-2 e^{\lambda-\nu} \omega \dot{\psi}_{2}=0
$$

and

$$
\psi_{2}^{\prime \prime}+\left(\frac{2}{r}+\frac{\nu^{\prime}-\lambda^{\prime}}{2}\right) \psi_{2}^{\prime}+e^{\lambda}\left(e^{-\nu} \omega^{2}-1\right) \psi_{2}-e^{\lambda-\nu} \ddot{\psi}_{2}+e^{\lambda-\nu} \frac{\dot{\nu}-\dot{\lambda}}{2}\left(\dot{\psi}_{2}-\omega \psi_{1}\right)+2 e^{\lambda-\nu} \omega \dot{\psi}_{1}=0 .
$$

Another equation we will find useful is $G_{\theta}^{\theta}=8 \pi G T_{\theta}^{\theta}$, which evaluates to

$$
\begin{aligned}
& e^{-\lambda}\left(\frac{\nu^{\prime}-\lambda^{\prime}}{2 r}+\frac{1}{2} \nu^{\prime \prime}+\frac{1}{4} \nu^{\prime 2}-\frac{1}{4} \nu^{\prime} \lambda^{\prime}\right)-e^{-\nu}\left(\frac{1}{2} \ddot{\lambda}+\frac{1}{4} \dot{\lambda}^{2}-\frac{1}{4} \dot{\nu} \dot{\lambda}\right) \\
& =e^{-\nu}\left(\dot{\phi}_{1}^{2}+\dot{\phi}_{2}^{2}+2 \omega\left(\dot{\phi}_{1} \psi_{2}-\dot{\phi}_{2} \psi_{1}\right)+\omega^{2}\left(\psi_{1}^{2}+\psi_{2}^{2}\right)\right)-e^{-\lambda}\left(\psi_{1}^{\prime 2}+\psi_{2}^{\prime 2}\right)-\left(\psi_{1}^{2}+\psi_{2}^{2}\right) .
\end{aligned}
$$

We use equations ( $\mathrm{C} 2$ ) through (C4) to obtain the equilibrium solutions, by setting

$$
\begin{aligned}
\lambda(t, r) & =\lambda_{0}(r) \\
\nu(t, r) & =\nu_{0}(r) \\
\psi_{1}(t, r) & =\phi_{0}(r) \\
\psi_{2}(t, r) & =0 .
\end{aligned}
$$

The equilibrium equations are then given by:

$$
\begin{gathered}
\lambda_{0}^{\prime}=\frac{1-e^{\lambda_{0}}}{r}+r\left[e^{\lambda_{0}}\left(\omega^{2} e^{-\nu_{0}}+1\right) \phi_{0}^{2}+\phi_{0}^{\prime 2}\right] \\
\nu_{0}^{\prime}=\frac{e^{\lambda_{0}}-1}{r}+r\left[e^{\lambda_{0}}\left(\omega^{2} e^{-\nu_{0}}-1\right) \phi_{0}^{2}+\phi_{0}^{\prime 2}\right] \\
\phi_{0}^{\prime \prime}=-\left(\frac{2}{r}+\frac{\nu_{0}^{\prime}-\lambda_{0}^{\prime}}{2}\right) \phi_{0}^{\prime}-e^{\lambda_{0}}\left(\omega^{2} e^{-\nu_{0}}-1\right) \phi_{0} .
\end{gathered}
$$

We now introduce four perturbation fields - $\delta \lambda(t, r), \delta \nu(t, r), \delta \psi_{1}(t, r)$ and $\delta \psi_{2}(t, r)$-and expand about the equilibrium configuration by writing:

$$
\begin{aligned}
\lambda(t, r) & =\lambda_{0}(r)+\delta \lambda(t, r) \\
\nu(t, r) & =\nu_{0}(r)+\delta \nu(t, r) \\
\psi_{1}(t, r) & =\phi_{0}(r)\left(1+\delta \psi_{1}(t, r)\right) \\
\psi_{2}(t, r) & =\phi_{0}(r) \delta \psi_{2}(t, r) .
\end{aligned}
$$

These last expressions are substituted into $(\overline{\mathrm{C} 2}),(\mathrm{C} 3),(\mathrm{C} 4)$ and $(\mathrm{C} 6)$ to obtain the following equations for the perturbed quantities: 


$$
\begin{aligned}
&\left(r e^{-\lambda_{0}} \delta \lambda\right)^{\prime}= r^{2}\left[2 \phi_{0}^{2} \delta \psi_{1}-e^{-\nu_{0}} \omega^{2} \phi_{0}^{2} \delta \nu+2 e^{-\nu_{0}} \omega^{2} \phi_{0}^{2} \delta \psi_{1}\right. \\
&\left.-2 e^{-\nu_{0}} \omega \phi_{0}^{2} \delta \dot{\psi}_{2}+2 e^{-\lambda_{0}} \phi_{0}^{\prime}\left(\phi_{0}^{\prime} \delta \psi_{1}+\phi_{0} \delta \psi_{1}^{\prime}\right)-e^{-\lambda_{0}} \phi_{0}^{\prime 2} \delta \lambda\right] \\
& \delta \nu^{\prime}-\delta \lambda^{\prime}=\left(\nu_{0}^{\prime}-\lambda_{0}^{\prime}+\frac{2}{r}\right) \delta \lambda-4 r e^{\lambda_{0}} \phi_{0}^{2} \delta \psi_{1} \\
& \delta \psi_{1}^{\prime \prime}+\left(\frac{2}{r}+\frac{\nu_{0}^{\prime}-\lambda_{0}^{\prime}}{2}+2 \frac{\phi_{0}^{\prime}}{\phi_{0}}\right) \delta \psi_{1}^{\prime}+\frac{\phi_{0}^{\prime}}{\phi_{0}}\left(\frac{\delta \nu^{\prime}-\delta \lambda^{\prime}}{2}\right) \\
&+e^{\lambda_{0}}\left(\omega^{2} e^{-\nu_{0}}-1\right) \delta \lambda-e^{\lambda_{0}-\nu_{0}} \omega^{2} \delta \nu-e^{\lambda_{0}-\nu_{0}} \delta \ddot{\psi}_{1}-2 e^{\lambda_{0}-\nu_{0}} \omega \delta \dot{\psi}_{2}=0 \\
& \quad-\delta \lambda e^{-\lambda_{0}}\left(\frac{\nu_{0}^{\prime}-\lambda_{0}^{\prime}}{2 r}+\frac{1}{2} \nu_{0}^{\prime \prime}+\frac{1}{4} \nu_{0}^{\prime 2}-\frac{1}{4} \nu_{0}^{\prime} \lambda_{0}^{\prime}\right) \\
&+e^{-\lambda_{0}}\left(\frac{\delta \nu^{\prime}-\delta \lambda^{\prime}}{2 r}+\frac{1}{2} \delta \nu^{\prime \prime}+\frac{1}{2} \nu_{0}^{\prime} \delta \nu^{\prime}-\frac{1}{4} \nu_{0}^{\prime} \delta \lambda^{\prime}-\frac{1}{4} \lambda_{0}^{\prime} \delta \nu^{\prime}\right)-\frac{1}{2} e^{-\nu_{0}} \delta \ddot{\lambda} \\
&=-\left[e^{-\nu_{0}} \omega^{2} \phi_{0}^{2} \delta \nu-e^{-\nu_{0}}\left(-2 \omega \phi_{0}^{2} \delta \dot{\psi}_{2}+2 \omega^{2} \phi_{0}^{2} \delta \psi_{1}\right)-e^{-\lambda_{0}} \phi_{0}^{\prime 2} \delta \lambda\right. \\
&+\left.e^{-\lambda_{0}}\left(2 \phi_{0}^{\prime 2} \delta \psi_{1}+2 \phi_{0} \phi_{0}^{\prime} \delta \psi_{1}^{\prime}\right)+2 \phi_{0}^{2} \delta \psi_{1}\right]
\end{aligned}
$$

The four equations above can be manipulated such that two variables, $\delta \nu$ and $\delta \psi_{2}$ are eliminated, leaving us with only two equations in two unknowns. To obtain the first of these two equations, we subtract (C18) from (C20) to get

$$
\begin{aligned}
\delta \psi_{1}^{\prime \prime}= & -\left(\frac{2}{r}+\frac{\nu_{0}^{\prime}-\lambda_{0}^{\prime}}{2}\right) \delta \psi_{1}^{\prime}-\frac{\delta \lambda^{\prime}}{r \phi_{0}^{2}}+e^{\lambda_{0}-\nu_{0}} \delta \ddot{\psi}_{1} \\
& -\left[\frac{\phi_{0}^{\prime}}{\phi_{0}}\left(\frac{\nu_{0}^{\prime}-\lambda_{0}^{\prime}}{2}+\frac{1}{r}\right)+\left(\frac{\phi_{0}^{\prime}}{\phi_{0}}\right)^{2}+\frac{1-r \lambda_{0}^{\prime}}{r^{2} \phi_{0}^{2}}+e^{\lambda_{0}-\nu_{0}} \omega^{2}-e^{\lambda_{0}}\right] \delta \lambda \\
& +2 e^{\lambda_{0}}\left[1+e^{-\nu_{0}} \omega^{2}+e^{-\lambda_{0}}\left(\frac{\phi_{0}^{\prime}}{\phi_{0}}\right)^{2}+r \phi_{0} \phi_{0}^{\prime}\right] \delta \psi_{1} .
\end{aligned}
$$

To obtain the other equation, we differentiate $(\overline{\mathrm{C} 19})$ with respect to $r$, and substitute the resulting expression, along with (C18) and (C19), into (C21) to get

$$
\begin{aligned}
\delta \lambda^{\prime \prime}= & -\frac{3}{2}\left(\nu_{0}^{\prime}-\lambda_{0}^{\prime}\right) \delta \lambda^{\prime}+\left[4 \phi_{0}^{\prime 2}+\lambda_{0}^{\prime \prime}+\frac{2}{r^{2}}-\frac{\left(\nu_{0}^{\prime}-\lambda_{0}^{\prime}\right)^{2}}{2}-\frac{2 \nu_{0}^{\prime}+\lambda_{0}^{\prime}}{r}\right] \delta \lambda+e^{\lambda_{0}-\nu_{0}} \delta \ddot{\lambda} \\
& -4\left(2 \phi_{0} \phi_{0}^{\prime}-r e^{\lambda_{0}} \phi_{0}^{2}\right) \delta \psi_{1}^{\prime} \\
& -4\left[2 \phi_{0}^{\prime 2}-r e^{\lambda_{0}} \phi_{0}^{2}\left(2 \frac{\phi_{0}^{\prime}}{\phi_{0}}+\frac{2 \nu_{0}^{\prime}+\lambda_{0}^{\prime}}{2}\right)\right] \delta \psi_{1},
\end{aligned}
$$

where, differentiating (C11) with respect to $r$ we have

$$
\begin{aligned}
\lambda_{0}^{\prime \prime} & =\frac{e^{\lambda_{0}}-1}{r^{2}}-\frac{e^{\lambda_{0}} \lambda_{0}^{\prime}}{r}+\left[e^{\lambda_{0}}\left(\omega^{2} e^{-\nu_{0}}+1\right) \phi_{0}^{2}+\phi_{0}^{\prime 2}\right] \\
& +r\left[-\nu_{0}^{\prime} \omega^{2} e^{\lambda_{0}-\nu_{0}} \phi_{0}^{2}+e^{\lambda_{0}}\left(\omega^{2} e^{-\nu_{0}}+1\right)\left(\lambda_{0}^{\prime} \phi_{0}^{2}+2 \phi_{0} \phi_{0}^{\prime}\right)+2 \phi_{0}^{\prime} \phi_{0}^{\prime \prime}\right] .
\end{aligned}
$$

(Note that ( $\mathrm{C22})$ omits a factor of $\exp \left(\lambda_{0}\right)$ which one finds in the $\sim \delta \lambda /\left(r^{2} \phi_{0}^{2}\right)$ term of equation (34) in [16].) For the stability analysis, we assume a harmonic time dependence, i.e.,

$$
\begin{array}{r}
\delta \psi_{1}(t, r)=\delta \psi_{1}(r) e^{i \sigma t} \\
\delta \lambda(t, r)=\delta \lambda(r) e^{i \sigma t} .
\end{array}
$$

Note that $(\overline{\mathrm{C} 23})$ and $(\overline{\mathrm{C} 24})$ contain only second derivatives with respect to time. There are good arguments for assuming $\sigma^{2}$ is purely real [14, 16], so we can determine instability by simply looking for instances where $\sigma^{2}<0$. 
As a further consideration, we note that the boson star system admits a conserved Noether current,

$$
J^{\mu}=\frac{i}{8 \pi} g^{\mu \nu}\left(\phi \partial_{\nu} \phi^{*}-\phi^{*} \partial_{\nu} \phi\right),
$$

for which the corresponding charge or "particle number" is

$$
\begin{aligned}
N & =\int d^{3} x \sqrt{-g} J^{t} \\
& =\int_{0}^{\infty} d r r^{2} e^{(\lambda-\nu) / 2}\left(\dot{\psi}_{1} \psi_{2}-\dot{\psi}_{2} \psi_{1}+\omega\left(\psi_{1}^{2}+\psi_{2}^{2}\right)\right) .
\end{aligned}
$$

Conventional stability analysis (see, e.g., [32]) demands that we consider only perturbations for which the total charge is conserved. Thus we compute the variation in the charge, $\delta N$, and work to ensure $\delta N=0$. In practice, since we cut off the grid at finite radius, it makes sense to consider the function $\delta N(r)$, the total charge enclosed in a sphere with surface area $4 \pi r^{2}$. This quantity is

$$
\begin{aligned}
\delta N(r) & =\frac{1}{\omega} \int_{0}^{r} d \tilde{r} \tilde{r}^{2} e^{\left(\nu_{0}-\lambda_{0}\right) / 2} \phi_{0}^{2} \\
& \times\left\{\frac{\delta \lambda^{\prime}}{2 \tilde{r} \phi_{0}^{2}}+\frac{1}{2}\left[e^{\lambda_{0}-\nu_{0}} \omega^{2}+\left(\frac{\phi_{0}^{\prime}}{\phi_{0}}\right)^{2}+\frac{1-\tilde{r} \lambda_{0}^{\prime}}{\tilde{r}^{2} \phi_{0}^{2}}\right] \delta \lambda\right. \\
& \left.-\frac{\phi_{0}^{\prime}}{\phi_{0}} \delta \psi_{1}^{\prime}-\left[-e^{\lambda_{0}-\nu_{0}} \omega^{2}+\left(\frac{\phi_{0}^{\prime}}{\phi_{0}}\right)^{2}+e^{\lambda_{0}}\right] \delta \psi_{1}\right\}
\end{aligned}
$$

where primes denote $\partial / \partial \tilde{r}$. (Note that C27) contains a term involving $\delta \psi_{1}^{\prime}$, which was not included in equation (35) of [16].) We then demand that $\delta N \rightarrow 0$ as $r \rightarrow \infty$.

The boundary conditions are as follows:

At $r=0$ :

$$
\begin{aligned}
\lambda_{0} & =0 \\
\nu_{0} & =0 \\
\phi_{0}^{\prime} & =0 \\
\phi_{0}^{\prime \prime} & =-\frac{1}{3}\left(\omega^{2}-1\right) \phi_{0} \\
\delta \psi_{1}^{\prime \prime} & =\frac{1}{3}\left[-\frac{3 \delta \lambda^{\prime \prime}}{2 \phi_{0}^{2}}+\left(2\left(\omega^{2}+1\right)-\sigma^{2}\right) \delta \psi_{1}\right] \\
\delta \lambda & =0 \\
\delta \lambda^{\prime} & =0 .
\end{aligned}
$$

As $r \rightarrow \infty:$

$$
\begin{gathered}
\delta N \rightarrow 0 \\
\delta \psi_{1} \rightarrow 0 \\
\delta \lambda \rightarrow 0
\end{gathered}
$$

To solve the system (C23) and (C24) subject to the above boundary conditions, for a given value of $\phi_{0}(0)$, we resort to the method of "shooting," first for the equilibrium solutions, then for the perturbed quantities. Specifically, we choose a value for $\omega$ and solve the equilibrium equations numerically by integrating outward from $r=0$. We do this repeatedly, performing a "binary search" on $\omega$ (as described in [12]) until the boundary conditions for the equilibrium quantities are satisfied.

Due to the linearity of the problem, we can choose $\delta \psi_{1}(0)$ arbitrarily. We then have two parameters left, namely $\sigma^{2}$ and $\delta \lambda^{\prime \prime}(0)$. To make matters easy at first, we consider perturbations very close to the transition between stability and instability. At the transition point, $\sigma^{2}$ is zero. Thus for boson stars near the transition point, we choose $\sigma^{2}=0$ and shoot on the parameter $\delta \lambda^{\prime \prime}(0)$ until the boundary conditions are satisfied. As Gleiser and Watkins [16] note, the transition point occurs at the maximum boson star mass; so we can take two slightly different equilibrium solutions near the maximum mass and subtract them to generate solutions which should agree with those obtained from the 
perturbation problem. We use this method to obtain a trial value of $\delta \lambda^{\prime \prime}(0)$, and also as a way of checking the final solution we obtain from the perturbation analysis.

For more general configurations $\left(\sigma^{2} \neq 0\right)$, we choose a value of $\sigma^{2}$ and shoot on $\delta \lambda^{\prime \prime}(0)$ until we find $\delta N$ at the outer boundary of the grid to be less than some tolerance value. Then we use the fact (gleaned from experience) that if $\sigma^{2}$ is too large (too positive), $\delta N$ will have a local minimum, the value of which will be less than zero (i.e., $\delta N(r)$ will dip below zero and then turn back up at larger radii). If $\sigma^{2}$ is too low there will be no such local minimum. We use these two criteria to select the value of $\sigma^{2}$ via a binary search. Thus our two-dimensional eigenvalue-finding algorithm consists simply of two (nested) binary searches, one in each direction: For each value of $\sigma^{2}$ tried, a full binary search on the parameter $\delta \lambda^{\prime \prime}(0)$ is performed to drive $\delta N\left(r_{\max }\right) \rightarrow 0$. Then the solution of $\delta N(r)$ is examined for the behavior described above, and a new value of $\sigma^{2}$ is selected, and so on until both $\delta \lambda^{\prime \prime}(0)$ and $\sigma^{2}$ have been found to some desired precision.

[1] M.W. Choptuik, Phys. Rev. Lett. 709 (1993).

[2] C. Gundlach, Adv. Theor. Math.Phys. 2 1-49 (1998).

[3] C. Gundlach, Living Reviews 1999-4 (1999).

[4] C.R. Evans and J.S. Coleman, Phys. Rev. Lett. 721782 (1994) .

[5] T. Koike, T. Hara and S. Adachi, Phys Rev. Lett. 745170 (1995).

[6] D.W. Neilsen and M.W. Choptuik, Class.Quant.Grav. 17761 -782 (2000).

[7] M.W. Choptuik, T. Chmaj and P. Bizon, Phys. Rev. Lett. 77 (424).

[8] P.R. Brady, C.M. Chambers and S.M.C.V. Conçalves, Phys. Rev. D 56, 6057 (1997).

[9] A.M. Abrahams and C.R. Evans, Phys. Rev. Lett. 702980 (1993).

[10] Studies involving Brill waves in three dimensions have demonstrated the existence of super-critical and sub-critical solutions, however isolation and study of the critical solutions per se will require considerably more resolution than that used in those studies. See M. Alcubierre, G. Allen, B. Bruegmann, G. Lanfermann, E. Seidel, W.-M. Suen, and M. Tobias, Phys. Rev. D61 041501 (2000), as well as T. Baumgarte, http://online.itp.ucsb.edu/online/numrel00/baumgarte/.

[11] D.J. Kaup, Phys. Rev. 1721331 (1968).

[12] R. Ruffini and S. Bonnazzola, Phys. Rev. 1871767 (1969).

[13] M. Colpi, S.L. Shapiro, and I. Wasserman, Phys. Rev. Lett. 572485 (1986).

[14] P. Jetzer, Phys. Rep. 220, 163 (1992).

[15] E.W. Mielke and F.E. Schunck. gr-qc/9801063.

[16] M. Gleiser and R. Watkins, Nucl. Phys. B319 733 (1989).

[17] T.D. Lee and Y. Pang, Nucl. Phys. B315 477 (1989).

[18] E. Seidel and W.-M. Suen, Phys. Rev. D42 384 (1990).

[19] E. Seidel and W.-M. Suen, Phys. Rev. Lett. 66, (1991).

[20] J. Balakrishna, E. Seidel and W.-M. Suen, Phys.Rev. D58 104004 (1998).

[21] F.E. Schunck and D.F. Torres, gr-qc/9911038.

[22] R. Arnowitt, S. Deser and C.W. Misner, in Gravitation: An Introduction to Current Research, ed. L. Witten, Wiley, New York (1962).

[23] E. Seidel and W.-M. Suen, Phys. Rev. Lett. 72, (1994).

[24] C. Gundlach and J.M. Martin-Garcia, Phys. Rev. D54, 7353 (1996).

[25] H.O. Kreiss and J. Oliger, Methods for the Approximate Solution of Time Dependent Problems, GARP Publications Series No. 10 (1973).

[26] M.W. Choptuik, A Study of Numerical Techniques for Radiative Problems in General Relativity, Ph.D. dissertation, University of British Columbia (1986).

[27] M. Israeli and S. Orszag, J. Comp. Phys. 41115 (1981).

[28] A. Arbona and C. Bona, Comput. Phys. Commun. 118229 (1999).

[29] A collection of MPEG movies showing the effects of various outer boundary conditions can be found at http://wwwrel.ph.utexas.edu/ shawley/bc.html

[30] E. Honda and M.W. Choptuik. In preparation.

[31] M.W. Choptuik, ad: An Implementation of the Berger-Oliger Mesh Refinement Algorithm for the Wave Equation in Spherical Symmetry. University of Texas at Austin, Center for Relativity, Technical Report UTREL/TR94-001 (unpublished).

[32] S.L. Shapiro and S.A. Teukolsky, Black Holes, White Dwarfs and Neutron Stars, Wiley, New York, (1983).

[33] M. Colpi, S.L. Shapiro and S.A. Teukolsky, Astrophys. J., 369, 422 (1991).

[34] K. Sumiyoshi, S. Yamada, H. Suzuki, and W. Hillebrandt, Astron. and Astrophys., 334, 159 (1998). 